\title{
Bimetric QED
}

\author{
I.T. Drummond* \\ Department of Applied Mathematics and Theoretical Physics \\ Centre for Mathematical Sciences \\ Wilberforce Road \\ Cambridge \\ England, CB3 0WA
}

November 6, 2018

\begin{abstract}
We study, as a model of Lorentz symmetry breaking, the quantisation and renormalisation of an extension of QED in a flat spacetime where the photons and electrons propagate differently and do not share the same lightcone. We will refer to this model as Bimetric QED (BIMQED). As a preliminary we discuss the formulation of electrodynamics in a pre-metric formalism showing nevertheless that there is, on the basis of a simple criteron, a preferred metric. Arising from this choice of metric is a Weyl-like tensor (WLT). The Petrov classification of the WLT gives rise to a corresponding classification of Lorentz symmetry breaking. We do not impose any constraint on the strength of the symmetry breaking and are able to obtain explicit dispersion relations for photon propagation in each of the Petrov classes. The associated birefringence appears in some cases as two distinct polarisation dependent lightcones and in other cases as a a more complicated structure that cannot be disentangled in a simple way.

We show how in BIMQED the renormalisation procedure can, in addition to its effect on standard parameters such as charge and mass, force the renormalisation of the metrics and the WLT. Two particularly tractable cases are studied in detail for which we can obtain renormalisation group flows for the parameters of the model together with an analysis of fixed point structure. Of course these results are consistent with previous studies but we are not constrained to treat Lorentz symmetry breaking as necessarily weak. As we found in a previous study of a scalar field theory model an acceptable causal structure for the model imposes constraints on relationship between the various lightcones in BIMQED.
\end{abstract}

DAMTP-2016-26

*email: itd@damtp.cam.ac.uk 


\section{Introduction}

In a previous paper [1] we studied a scalar field theory model in which each field was associated with a distinct metric. For simplicity we assumed that spacetime was represented by a flat background and parametrised by coordinates $x^{\mu}$ such that the theory is invariant under the translations $x^{\mu} \rightarrow x^{\mu}+a^{\mu}$. The metrics are then independent of the coordinates $x^{\mu}$. We formulated the theory so that it was invariant under general linear transformations $x^{\mu} \rightarrow M^{\mu}{ }_{\nu} x^{\nu}$. The implications of the model were that in addition to a renormalisation of the coupling constants and masses such theories required a renormalisation of the metrics. An examination of the renormalisation group showed that the important effect was a renormalisation of the relationships between the metrics and their associated lightcones. These relationships are therefore dependent on the renormalisation scale $\mu$ (we use dimensional regularisation). We found that at each stage the associated lightcones must overlap by sharing some interior vectors that are timelike in all the metrics. This constraint on the lightcones originates in a requirement that the evolution of the full system of interacting quantum fields is causal for some set of observers. The renormalisation of the metric relationship will have implications for models in which two (or more) metrics become dynamical degrees of freedom.

Of course such models exhibit a breakdown of Lorentz invariance and correspond to a subset of $C P T$-even violations. A more general set of effects has been the focus of a wide range of investigations by many authors $[2,3,4,5,6,7]$. We believe however that our approach of concentrating on multimetric theories sheds further light on this particular sector of the violation of Lorentz invariance.

In this paper we consider a version of QED, Bimetric QED (BIMQED) that associates one metric with the electromagnetic and another with the electron field. A simple extension of the model could involve the introduction of a third metric associated with the muon field, though we do not pursue this here. Of course we do not see this model as any more than a demonstration of the ideas in a simple gauge theory since there is so far no observational reason for anticipating a breakdown of Lorentz invariance in QED. The same analysis applied to theories with non-abelian gauge groups is also of interest especially in relation to high energy scattering. In fact the model is related to, but in some ways simpler than, those investigated by Nielsen and Ninomiya [8], and subsequently by Nielsen and Picek [9] and Chadha and Nielsen [10].

Our starting point is a formulation of electrodynamics that has been referred to as "premetric" [11]. We examine this formulation and show that in fact there is a preferred metric. Identifying this metric also permits a clear statement of the nature of Lorentz symmetry breaking for electrodynamics. The breaking of Lorentz symmetry is associated with a tensor that has the symmetry properties of the Weyl tensor in general relativity. We refer to this as a Weyl-like tensor (WLT). The Petrov classification [12] for such tensors can be used to identify the different kinds of symmetry breaking that are possible. Of course this analysis is consistent with other work $[13,14,15]$ on the breaking of Lorentz symmetry in electrodynamics. A feature of our approach is that while we do use the standard perturbation expansion in electric charge we are not constrained to treat Lorentz symmetry breaking perturbatively, unless this happens to be convenient.

\section{Preferred Metric in Electrodynamics}

The pre-metric formulation of electrodynamics [11] in its most general form replaces Maxwell's equations for the gauge field $A_{\mu}(x)$ with a modified set of the form

$$
\partial_{\mu} \tilde{U}^{\mu \nu \sigma \tau} F_{\sigma \tau}(x)=0,
$$

where

$$
F_{\sigma \tau}(x)=\partial_{\sigma} A_{\tau}(x)-\partial_{\tau} A_{\sigma}(x),
$$

and the (constant) tensor density $\tilde{U}^{\mu \nu \sigma \tau}$ satifies

$$
\tilde{U}^{\mu \nu \sigma \tau}=-\tilde{U}^{\nu \mu \sigma \tau}=-\tilde{U}^{\mu \nu \tau \sigma} .
$$


In this general formulation there is no requirement that $\tilde{U}^{\mu \nu \sigma \tau}=\tilde{U}^{\sigma \tau \mu \nu}$. Indeed the nonvanishing of the antisymmetric contribution $\tilde{U}^{\mu \nu \sigma \tau}-\tilde{U}^{\sigma \tau \mu \nu}$ produces what are referred to as skewon effectsi [11]. Since however we wish to derive our dynamical equations from a Lagrangian formulation we will exclude skewon effects. We will also introduce a (constant) metric $g_{\mu \nu}$ and set

$$
\tilde{U}^{\mu \nu \sigma \tau}=\Omega U^{\mu \nu \sigma \tau}
$$

where

$$
\operatorname{det} g_{\mu \nu}=-\Omega^{2}
$$

and the tensor $U^{\mu \nu \sigma \tau}$ satisfies

$$
\begin{aligned}
& U^{\mu \nu \sigma \tau}=-U^{\nu \mu \sigma \tau}, \\
& U^{\mu \nu \sigma \tau}=-U^{\mu \nu \tau \sigma}, \\
& U^{\mu \nu \sigma \tau}=U^{\sigma \tau \mu \nu},
\end{aligned}
$$

together with

$$
U^{\mu \nu \sigma \tau}+U^{\mu \sigma \tau \nu}+U^{\mu \tau \nu \sigma}=0 .
$$

As remarked by Nielsen and Ninomiya [8], this gives $U^{\mu \nu \sigma \tau}$ the algebraic properties of the Riemann tensor although there is no necessary connection. However see ref $[16,17,18,19]$. For the given metric there is a standard decomposition of $U^{\mu \nu \sigma \tau}$ in the form

$$
U^{\mu \nu \sigma \tau}=\frac{1}{12} U\left(g^{\mu \sigma} g^{\nu \tau}-g^{\mu \tau} g^{\nu \sigma}\right)+\frac{1}{2}\left(g^{\mu \sigma} S^{\nu \tau}+S^{\mu \sigma} g^{\nu \tau}-g^{\mu \tau} S^{\nu \sigma}-S^{\mu \tau} g^{\nu \sigma}\right)-C^{\mu \nu \sigma \tau},
$$

where

$$
\begin{aligned}
S^{\mu \sigma} & =U^{\mu \sigma}-\frac{1}{4} U g^{\mu \sigma} \\
U^{\mu \sigma} & =g_{\nu \tau} U^{\mu \nu \sigma \tau} \\
U & =g_{\mu \sigma} U^{\mu \sigma}
\end{aligned}
$$

and

$$
g_{\nu \tau} C^{\mu \nu \sigma \tau}=0
$$

Clearly $g_{\mu \sigma} S^{\mu \sigma}=0$ and $C^{\mu \nu \sigma \tau}$ has the algebraic properties of the Weyl tensor.

So far the metric is arbitrary. We can arrive at a preferred metric by demanding that the tensor $U^{\mu \nu \sigma \tau}$ is most nearly like $g^{\mu \sigma} g^{\nu \tau}-g^{\mu \tau} g^{\nu \sigma}$. We implement this idea by requiring that the overlap amplitude $U^{\mu \nu \sigma \tau}\left(g_{\mu \sigma} g_{\nu \tau}-g_{\mu \tau} g_{\nu \sigma}\right)$ is stationary with respect to variations of $g_{\mu \sigma}$ subject to the constraint in eq(5). Introducing the Lagrange multiplier $\lambda$ and setting

$$
\mathcal{F}=U^{\mu \nu \sigma \tau}\left(g_{\mu \sigma} g_{\nu \tau}-g_{\mu \tau} g_{\nu \sigma}\right)-\lambda \operatorname{det} g_{\mu \sigma}
$$

we require that

$$
\frac{\partial \mathcal{F}}{g_{\mu \sigma}}=0 .
$$

This yields

$$
U^{\mu \sigma}+\lambda \Omega^{2} g^{\mu \sigma}=0 .
$$

It follows that for this stationary value of the metric that

$$
S^{\mu \sigma}=0 .
$$

Of course we are assuming that $U^{\mu \nu \sigma \tau}$ is such that it yields a unique solution of eq(13) with the right type of (lightcone generating) metric.

The action for the elctromagnetic field that gives rise to eq(1) is $S_{(p)}$ where

$$
S_{(p)}=-\frac{1}{8} \int d^{4} x \Omega U^{\mu \nu \sigma \tau} F_{\mu \nu}(x) F_{\sigma \tau}(x)
$$


For a given metric we are free to adjust the normalisation of the field $A_{\mu}(x)$ so that the normalisation of $U^{\mu \nu \sigma \tau}$ is such that $U=12$. We have then $S_{(p)}$ is given by eq(15) where

$$
U^{\mu \nu \sigma \tau}=\left(g^{\mu \sigma} g^{\nu \tau}-g^{\mu \tau} g^{\nu \sigma}\right)-C^{\mu \nu \sigma \tau} .
$$

It follows that when the Weyl-like tensor $C^{\mu \nu \sigma \tau}$ vanishes $S_{\gamma}$ is invariant under the Lorentz group that leaves $g_{\mu \nu}$ invariant and there is no birefringence in the evolution of the elctromagnetic field. However when $C^{\mu \nu \sigma \tau}$ is non-vanishing we do encounter birefringence and the breaking of Lorentz invariance. The possible ways in which Lorentz symmetry breaking occurs can therefore be given a Petrov classification appropriate to the tensor $C^{\mu \nu \sigma \tau}$.

\section{Equations of Motion}

The gauge invariant equations of motion, eq(1), now take the form

$$
\left(g^{\mu \sigma} g^{\nu \tau}-g^{\mu \tau} g^{\nu \sigma}-C^{\mu \nu \sigma \tau}\right) \partial_{\sigma} \partial_{\mu} A_{\nu}(x)=0 .
$$

This is the standard form with Lorentz symmetry breaking for electrodynamics. Our analysis makes clear that the there is no lack of generality in this form and that we can always choose the preferred metric for which the Lorentz symmetry breaking is described by the traceless WLT $C^{\mu \nu \sigma \tau}$.

If we seek a solution of the form

$$
A_{\nu}(x)=\varepsilon_{\nu} e^{-i q \cdot x}
$$

then $\varepsilon_{\nu}$ satisfies

$$
M^{\tau \nu} \varepsilon_{\nu}=0
$$

where

$$
M^{\tau \nu}=q^{2} g^{\tau \nu}-q^{\tau} q^{\nu}-C^{\mu \nu \sigma \tau} q_{\mu} q_{\sigma},
$$

and we set $q^{\mu}=g^{\mu \nu} q_{\nu}$. Of course eq(19) has the solution $\varepsilon_{\mu} \propto q_{\mu}$ that for any vlaue of $q_{\mu}$. It corresponds to a gauge degree of freedom. The physical solutions appear only when $q_{\mu}$ is constrained to satisfy a dispersion relation that permits the rank of the matrix $M^{\tau \nu}$ to drop below the value 3 and its kernel to have a dimension greater than 1. See also ref [11].

Following conventional lines of reasoning we can explore plane wave solutions by imposing the gauge condition

$$
q^{\nu} \varepsilon_{\nu}=0 .
$$

$\mathrm{Eq}(19)$ becomes

$$
\left\{q^{2} g^{\tau \nu}-C^{\mu \nu \sigma \tau} q_{\mu} q_{\sigma}\right\} \varepsilon_{\nu}=0 .
$$

It is easy to see that eq(22) implies

$$
q^{2} q^{\nu} \varepsilon_{\nu}=0 .
$$

Hence eq(21) can be imposed in a consistent manner. The problem then reduces to finding the conditions on $q_{\mu}$ that allow eq(22) to have nontrivial solutions. We will return to the issue of gauge conditions later.

\subsection{Newman-Penrose Tetrad}

It is convenient to reformulate these equations of motion in terms of a Newman-Penrose tetrad $[20,21]$. It comprises four null vectors, $l_{\mu}, n_{\mu}, m_{\mu}$ and $\bar{m}_{\mu}$ where $l_{\mu}$ and $n_{\mu}$ are real and $m_{\mu}$ and $\bar{m}_{\mu}$ are complex conjugates. They satisfy the relations

$$
l^{\mu} l_{\mu}=n^{\mu} n_{\mu}=m^{\mu} m_{\mu}=\bar{m}^{\mu} \bar{m}_{\mu}=0,
$$

and

$$
l^{\mu} m_{\mu}=l^{\mu} \bar{m}_{\mu}=n^{\mu} m_{\mu}=n^{\mu} \bar{m}_{\mu}=0
$$


together with

$$
l^{\mu} n_{\mu}=-m^{\mu} \bar{m}_{\mu}=1
$$

We have also

$$
g^{\mu \nu}=l^{\mu} n^{\nu}+n^{\mu} l^{\nu}-m^{\mu} \bar{m}^{\nu}-\bar{m}^{\mu} m^{\nu} .
$$

Hence $\varepsilon$ can be re-expressed in terms of its components in the NP tetrad basis,

$$
\varepsilon^{\mu}=l^{\mu}(n . \varepsilon)+n^{\mu}(l . \varepsilon)-m^{\mu}(\bar{m} . \varepsilon)-\bar{m}^{\mu}(m . \varepsilon) .
$$

For later convenience we reformulate eq(22) also in the tetrad basis. Introduce $N^{\nu \tau}$ where

$$
N^{\nu \tau}=C^{\mu \nu \sigma \tau} q_{\mu} q_{\sigma}
$$

and the matrix entries $N_{l l}=N^{\nu \tau} l_{\nu} l_{\tau}, N_{l n}=N^{\nu \tau} l_{\nu} n_{\tau}$ etc. We have then

$$
\left(\begin{array}{cccc}
q^{2}-N_{l n} & -N_{l l} & N_{l \bar{m}} & N_{l m} \\
-N_{n n} & q^{2}-N_{n l} & N_{n \bar{m}} & N_{n m} \\
-N_{m n} & -N_{m l} & q^{2}+N_{m \bar{m}} & N_{m m} \\
-N_{\bar{m} n} & -N_{\bar{m} l} & N_{\bar{m} \bar{m}} & q^{2}+N_{\bar{m} m}
\end{array}\right)\left(\begin{array}{c}
l . \varepsilon \\
n . \varepsilon \\
m . \varepsilon \\
\bar{m} . \varepsilon
\end{array}\right)=0 .
$$

For non-trivial solutions we require the vanishing of the determinant of the matrix in eq(30). In examples below we will see that this determinant has a factor of $\left(q^{2}\right)^{2}$ corresponding to gauge modes. The remaining factor yields the lightcone structure of the physical modes.

\section{Petrov Classification of Lorentz Symmetry Breaking}

The Petrov classification of Weyl-like tensors (WLTs) [12] can be expressed in a number of ways. A powerful way of understanding the structure of WLTs is the Newman-Penrose (NP) formalism together with the Penrose spinor approach [22]. A succinct account of the this formalism is provided by Stewart [21]. A simple account may also be found in [23]. An important concept in relation to a WLT is that of a principal null direction (PND). Such a PND is represented by a null vector, $v_{\mu}$, that satisfies the constraint

$$
v_{[\alpha} C_{\mu] \nu \sigma[\tau} v_{\beta]} v^{\nu} v^{\sigma}=0 .
$$

In general there are four distinct directions that are solutions of this equation and the WLT is Petrov class I. When the constraint has one double root and there are three distinct PNDs the WLT is Petrov class II. The case of two double roots is Petrov class D. The PND corresponding to a double root in these two cases satisfies a modified (but consistent) constraint

$$
C_{\mu \nu \sigma[\tau} v_{\beta]} v^{\nu} v^{\sigma}=0 .
$$

When there is a triple root, and two distinct PNDs, the WLT is of Petrov class III and the PND corresponding to the triple root satisfies a further modified constraint

$$
C_{\mu \nu \sigma[\tau} v_{\beta]} v^{\sigma}=0 .
$$

Finally when all four roots coincide the WLT is of Petrov class N and the PND satisfies the constraint

$$
C_{\mu \nu \sigma \tau} v^{\sigma}=0 .
$$

The underlying algebra that supports these results together with further implications utilises the NP and spinor formalism that is explained in refs [20, 22, 21, 23]. 


\subsection{Canonical Forms for the Weyl-like Tensor}

It is useful for the purposes of explicit calculation to identify canonical forms associated with the Petrov classification of the WLTs. While these are not unique they may be expressed in terms of the NP tetrad. It is convenient to introduce the antisymmetric tensors, $A_{\mu \nu}, B_{\mu \nu}$ and $D_{\mu \nu}$ where

$$
\begin{aligned}
A_{\mu \nu} & =l_{\mu} m_{\nu}-l_{\nu} m_{\mu} \\
B_{\mu \nu} & =\bar{m}_{\mu} n_{\nu}-\bar{m}_{\nu} n_{\mu} \\
D_{\mu \nu} & =l_{\mu} n_{\nu}-l_{\nu} n_{\mu}+\bar{m}_{\mu} m_{\nu}-\bar{m}_{\nu} m_{\mu}
\end{aligned}
$$

For class $\mathrm{N}$ we can choose the single PND to be $l_{\mu}$ and the WLT to have the form

$$
C_{\mu \nu \sigma \tau}=A_{\mu \nu} A_{\sigma \tau}+\text { c.c. },
$$

where c.c. indicates complex conjugate. For class III we have

$$
C_{\mu \nu \sigma \tau}=A_{\mu \nu} D_{\sigma \tau}+D_{\mu \nu} A_{\sigma \tau}+\text { c.c.. }
$$

For class D

$$
C_{\mu \nu \sigma \tau}=\lambda\left\{A_{\mu \nu} B_{\sigma \tau}+B_{\mu \nu} A_{\sigma \tau}+D_{\mu \nu} D_{\sigma \tau}\right\}+\text { c.c.. }
$$

For class II

$$
C_{\mu \nu \sigma \tau}=\lambda\left\{A_{\mu \nu} A_{\sigma \tau}+\frac{1}{6}\left[A_{\mu \nu} B_{\sigma \tau}+B_{\mu \nu} A_{\sigma \tau}+D_{\mu \nu} D_{\sigma \tau}\right]\right\}+\text { c.c.. }
$$

For class I

$$
C_{\mu \nu \sigma \tau}=\mu\left\{A_{\mu \nu} A_{\sigma \tau}+B_{\mu \nu} B_{\sigma \tau}\right\}+\lambda\left\{A_{\mu \nu} B_{\sigma \tau}+B_{\mu \nu} A_{\sigma \tau}+D_{\mu \nu} D_{\sigma \tau}\right\}+\text { c.c.. }
$$

In classes I, II, and D, $\lambda$ and $\mu$ are complex parameters. In classes III and $\mathrm{N}$, any such complex parameter can be absorbed into the definition of $l$ and $m$ by subjecting them to an appropriate Lorentz transformation and rotation respectively. However in the context of renormalisation analysis this may not always be convenient. Where appropriate we will reinstate coefficients.

\subsection{Example for Petrov Class O}

The very simplest case of Petrov class $\mathrm{O}$, in which $C^{\mu \nu \sigma \tau}$ vanishes is not included in the above list. For a non-interacting elctromagnetic field it implies no Lorentz symmetry breakdown. However in the case of QED, Lorentz symmetry may be broken because of differing lightcone structure for the photons and the electrons without the introducion of a WLT into the dynamics. We will study such cases later in the context of renormalisation theory.

\subsection{Example for Petrov Class $\mathrm{N}$}

The simplest non-trivial case is Petrov class N. Eq(30) becomes

$$
\left(\begin{array}{cccc}
q^{2} & 0 & 0 & 0 \\
-(m . q)^{2}-(\bar{m} . q)^{2} & q^{2} & (l . q)(m . q) & (l . q)(\bar{m} . q) \\
-(l . q)(\bar{m} \cdot q) & 0 & q^{2} & (l . q)^{2} \\
-(l . q)(m . q) & 0 & (l . q)^{2} & q^{2}
\end{array}\right)\left(\begin{array}{c}
l . \varepsilon \\
n . \varepsilon \\
m \cdot \varepsilon \\
\bar{m} \cdot \varepsilon
\end{array}\right)=0 .
$$

It is easy to show that the determinant $\Delta$ of the matrix in eq(41) is given by

$$
\Delta=\left(q^{2}\right)^{2}\left(\left(q^{2}\right)^{2}-(l . q)^{4}\right) .
$$

We have then either

$$
q^{2}=0 \quad \text { (twice) }
$$




$$
q^{2}= \pm(l . q)^{2}
$$

When $q^{2}=0,(n . \varepsilon)$ is arbitrary and there remains the solution for which $\varepsilon \propto q$. The general solution is then

$$
\varepsilon_{\tau}=\alpha q_{\tau}+\beta l_{\tau}
$$

where $\alpha$ and $\beta$ are arbitrary parameters.

When $q^{2} \neq 0$ we find $l . \varepsilon=0$. Then we have

$$
\begin{aligned}
& q^{2} m . \varepsilon+(l . q)^{2} \bar{m} \cdot \varepsilon=0 \\
& (l . q)^{2} m . \varepsilon+q^{2} \bar{m} \cdot \varepsilon=0
\end{aligned}
$$

$\operatorname{Eq}(44)$ then allows non-trivial solutions $\varepsilon^{( \pm)}$which satisfy

$$
(m \pm \bar{m}) \cdot \varepsilon^{( \pm)}=0
$$

In the present example then we see that Lorentz symmetry breakdown is revealed by the birefringence associated with the two lightcones implicit in eq(44). The corresponding polarisation vectors are determined through eq(47) by the spatial axes $m$ and $\bar{m}$.

One further point of significance is that by making a Lorentz transformation of coordinates in the $l-n$ plane with a hyperbolic angle $\psi$, the PND vector becomes $e^{\psi} l_{\mu}$. Correspondingly

$n_{\mu} \rightarrow e^{-\psi} n_{\mu}$. The size of the components of $l_{\mu}$, as remarked above, can therefore be adjusted arbitrarily simply by making an appropriate choice of $\psi$. In a sense then, the same Lorentz symmetry breaking situation can be viewed as either large or small depending which coordinate basis is appropriate for describing the motion of the relevant observer.

\subsection{Example for Petrov Class III}

When the WLT is Petrov class III there are two PNDs, a triple root $l_{\mu}$ and a single root $n_{\mu}$. They can be embedded in the NP tetrad as above and used to construct the the WLT thus

$$
C_{\mu \nu \sigma \tau}=A_{\mu \nu} D_{\sigma \tau}+D_{\mu \nu} A_{\sigma \tau}+\text { c.c.. }
$$

We find that eq(30) becomes

$$
\mathcal{M}\left(\begin{array}{c}
l . \varepsilon \\
n . \varepsilon \\
m . \varepsilon \\
\bar{m} . \varepsilon
\end{array}\right)=0
$$

where the columns, $\mathcal{M}_{i}(i=1,2,3,4)$, of the matrix $\mathcal{M}$ are given by

$$
\begin{gathered}
\mathcal{M}_{1}=\left(\begin{array}{c}
q^{2}+l \cdot q m \cdot q+l \cdot q \bar{m} \cdot q \\
-2 n \cdot p m \cdot q-2 n \cdot q \bar{m} \cdot q \\
-l \cdot q n \cdot q-m \cdot q \bar{m} \cdot q+(m \cdot q)^{2} \\
-l \cdot q n \cdot q-m \cdot q \bar{m} \cdot q+(\bar{m} \cdot q)^{2}
\end{array}\right) \\
\mathcal{M}_{2}=\left(\begin{array}{c}
q^{2}+l \cdot q m \cdot q+l \cdot q \bar{m} \cdot q \\
(l \cdot q)^{2} \\
(l \cdot q)^{2} \\
-(l \cdot q)^{2}
\end{array}\right) \\
\mathcal{M}_{3}=\left(\begin{array}{c}
0 \\
m \cdot q \bar{m} \cdot q+l \cdot q n \cdot q-(\bar{m} \cdot q)^{2} \\
q^{2}-l \cdot q m \cdot q-l \cdot q \bar{m} \cdot q \\
2 l . q \bar{m} \cdot q
\end{array}\right)
\end{gathered}
$$




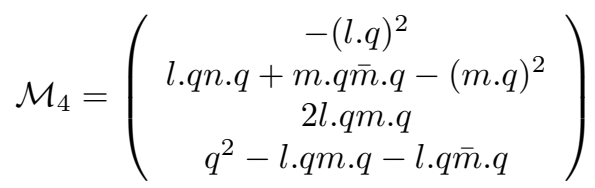

After some calculation the determinant of $\mathcal{M}$ can be obtained in the form

$$
\operatorname{det} \mathcal{M}=\left(q^{2}\right)^{2}\left[\left(q^{2}-(l . q)^{2}\right)^{2}-\left(4 l . q m . q-(l . q)^{2}\right)\left(4 l . q \bar{m} . q-(l . q)^{2}\right)\right] .
$$

As expected on general grounds, see ref [11], the second factor in eq(54) is a quartic expression the vanishing of which yields the dispersion relations for the two physical photon modes. There are two branches

$$
q^{2}-(l . q)^{2}= \pm(l . q) \sqrt{(4 m . q-l . q)(4 \bar{m} \cdot q-l . q)}
$$

However in contrast to the previous example for Petrov class $\mathrm{N}$, the quartic does not have quadratic factors. Therefore the birefringence in this case cannot be described by two distinct conventional lightcones. As is implicit in the derivation, the absolute strength of $C_{\mu \nu \sigma \tau}$ can can be adjusted by changing coordinates through Lorentz boosts in the $l_{\mu}-n_{\mu}$ plane and rotations in the $m-\bar{m}$ plane. In this way Petrov class III has features in common with class N.

\subsection{Example for Petrov Class D}

When the WLT is Petrov class $\mathrm{D}$, there are again two PNDs, $l_{\mu}$ and $n_{\mu}$ both being double roots. They can be embedded in the NP tetrad and yield a WLT of the form

$$
C_{\mu \nu \sigma \tau}=\lambda\left\{A_{\mu \nu} B_{\sigma \tau}+B_{\mu \nu} A_{\sigma \tau}+D_{\mu \nu} D_{\sigma \tau}\right\}+\text { c.c.. }
$$

We find that eq(30) takes the form eq(49) where the columns of $\mathcal{M}$ are (we denote the complex conjugate of $\lambda$ by $\bar{\lambda}$ )

$$
\begin{aligned}
& \mathcal{M}_{1}=\left(\begin{array}{c}
q^{2}+(\lambda+\bar{\lambda})(l \cdot q n \cdot q+m \cdot q \bar{m} \cdot q) \\
-(\lambda+\bar{\lambda})(n \cdot q)^{2} \\
(2 \lambda-\bar{\lambda}) n \cdot q m . q \\
(2 \bar{\lambda}-\lambda) n \cdot q \bar{m} \cdot q
\end{array}\right) \\
& \mathcal{M}_{2}=\left(\begin{array}{c}
-(\lambda+\bar{\lambda})(l . q)^{2} \\
q^{2}+(\lambda+\bar{\lambda})(l . q n . q+m . q \bar{m} . q) \\
(2 \bar{\lambda}-\lambda) l . q m \cdot q \\
(2 \lambda-\bar{\lambda}) l . q \bar{m} \cdot q
\end{array}\right) \\
& \mathcal{M}_{3}=\left(\begin{array}{c}
-(2 \lambda-\bar{\lambda}) l \cdot q \bar{m} \cdot q \\
-(2 \bar{\lambda}-\lambda) n \cdot q \bar{m} \cdot q \\
q^{2}-(\lambda+\bar{\lambda})(l \cdot q n \cdot q+m \cdot q \bar{m} \cdot q) \\
(\lambda+\bar{\lambda})(\bar{m} \cdot q)^{2}
\end{array}\right) \\
& \mathcal{M}_{4}=\left(\begin{array}{c}
-(2 \bar{\lambda}-\lambda) l . q m \cdot q \\
-(2 \lambda-\bar{\lambda}) n . q m \cdot q \\
(\lambda+\bar{\lambda})(m . q)^{2} \\
q^{2}-(\lambda+\bar{\lambda})(l . q n . q+m . q \bar{m} \cdot q)
\end{array}\right)
\end{aligned}
$$

In the present case the coefficient $\lambda$ cannot be absorbed by a redefinition of the vectors of the NP tetrad. The determinant of $\mathcal{M}$ can be obtained in the form

$$
\operatorname{det} \mathcal{M}=(1+\lambda+\bar{\lambda})\left(q^{2}\right)^{2}\left\{\left(q^{2}-(\lambda+\bar{\lambda}) l . q n . q+\rho m . q \bar{m} \cdot q\right)^{2}-9 \lambda \bar{\lambda}(1+\kappa)(1+\bar{\kappa})(m . q)^{2}(\bar{m} \cdot q)^{2}\right\},
$$

where the parameters $\kappa$ and $\rho$ are given by

$$
\kappa=\frac{1-(2 \lambda-\bar{\lambda})}{1+\lambda+\bar{\lambda}}
$$


and

$$
\rho=\lambda+\bar{\lambda}-\frac{9 \lambda \bar{\lambda}}{1+\lambda+\bar{\lambda}} .
$$

The dispersion relation for the physical modes implied by the vanishing of $\operatorname{det} \mathcal{M}$ does factorise in this case and yields two distinct light cones each with a dispersion relation that is quadratic in $q$,

$$
q^{2}-(\lambda+\bar{\lambda}) l . q n \cdot q+\rho m \cdot q \bar{m} \cdot q= \pm 3 \sqrt{\lambda \bar{\lambda}(1+\kappa)(1+\bar{\kappa})} m \cdot q \bar{m} \cdot q .
$$

\subsection{Example for Petrov Class II}

When the WLT is Petrov class II, there are three PNDs. In terms of the basis of the NP tetrad the double root is $l_{\mu}$ and the two single roots are $l_{\mu}+n_{\mu} \mp i\left(m_{\mu}-\bar{m}_{\mu}\right)$. The WLT has the form

$$
C_{\mu \nu \sigma \tau}=\lambda\left\{A_{\mu \nu} A_{\sigma \tau}+A_{\mu \nu} A_{\sigma \tau}+\frac{1}{6}\left[A_{\mu \nu} B_{\sigma \tau}+B_{\mu \nu} A_{\sigma \tau}{ }^{6}+D_{\mu \nu} D_{\sigma \tau}\right]\right\}+\text { c.c. }
$$

We find that eq(30) takes the form eq(41) where the columns of $\mathcal{M}$ are

$$
\begin{aligned}
& \mathcal{M}_{1}=\left(\begin{array}{c}
q^{2}+\frac{1}{6}(\lambda+\bar{\lambda})(l \cdot q n \cdot q+m \cdot q \bar{m} \cdot q) \\
-\lambda(m \cdot q)^{2}-\bar{\lambda}(\bar{m} \cdot q)^{2}-\frac{1}{6}(\lambda+\bar{\lambda})(n \cdot q)^{2} \\
-\bar{\lambda} l \cdot q \bar{m} \cdot q+\frac{1}{6}(2 \lambda-\bar{\lambda}) n \cdot q m \cdot q \\
-\lambda l \cdot q m \cdot q+\frac{1}{6}(2 \bar{\lambda}-\lambda) n \cdot q \bar{m} \cdot q
\end{array}\right) \\
& \mathcal{M}_{2}=\left(\begin{array}{c}
-\frac{1}{6}(\lambda+\bar{\lambda})(l . q)^{2} \\
q^{2}+\frac{1}{6}(\lambda+\bar{\lambda})(l \cdot q n . q+m . q \bar{m} . q) \\
\frac{1}{6}(2 \bar{\lambda}-\lambda) \text { l.qm.q } \\
\frac{1}{6}(2 \lambda-\bar{\lambda}) \text { l.qm.q }
\end{array}\right) \\
& \mathcal{M}_{3}=\left(\begin{array}{c}
-\frac{1}{6}(2 \lambda-\bar{\lambda}) l . q \bar{m} \cdot q \\
\lambda l . q m \cdot q-\frac{1}{6}(2 \bar{\lambda}-\lambda) n \cdot q \bar{m} \cdot q \\
q^{2}-\frac{1}{6}(\lambda+\bar{\lambda})(l \cdot q n \cdot q+m . q \bar{m} \cdot q) \\
\lambda(l . q)^{2}+\frac{1}{6}(\lambda+\bar{\lambda})(\bar{m} \cdot q)^{2}
\end{array}\right) \\
& \mathcal{M}_{4}=\left(\begin{array}{c}
-\frac{1}{6}(2 \bar{\lambda}-\lambda) l . q m . q \\
\bar{\lambda} l . q \bar{m} \cdot q-\frac{1}{6}(2 \lambda-\bar{\lambda}) n . q m . q \\
\bar{\lambda}(l . q)^{2}+\frac{1}{6}(\lambda+\bar{\lambda})(m . q)^{2} \\
q^{2}-\frac{1}{6}(\lambda+\bar{\lambda})(l . q n . q+m . q \bar{m} . q)
\end{array}\right)
\end{aligned}
$$

We find that

$$
\begin{aligned}
\operatorname{det} \mathcal{M}=\left[\left(1+\frac{1}{6}(\lambda+\bar{\lambda})\right]\left(q^{2}\right)^{2}\{\right. & {\left[\left(1-\frac{1}{12}(\lambda+\bar{\lambda})\right) q^{2}+\frac{1}{2} \bar{\lambda}(\kappa-1) m \cdot q \bar{m} \cdot q\right] } \\
& \times\left[\left(1-\frac{1}{12}(\lambda+\bar{\lambda})\right) q^{2}+\frac{1}{2} \lambda(\bar{\kappa}-1) m \cdot q \bar{m} \cdot q\right] \\
-\left[\lambda(l . q)^{2}\right. & \left.\left.+\frac{1}{2} \lambda(\kappa+1)(\bar{m} \cdot q)^{2}\right]\left[\bar{\lambda}(l \cdot q)^{2}+\frac{1}{2} \bar{\lambda}(\bar{\kappa}+1)(m \cdot q)^{2}\right]\right\} .
\end{aligned}
$$

Here we have

$$
\kappa=\frac{1-(2 \lambda-\bar{\lambda}) / 6}{1+(\lambda+\bar{\lambda}) / 6}
$$

Clearly the quartic expression providing the dispersion relation for the physical modes again does not, in general, factorise into separate quadratic factors so the lightcone structure is more complex than two separate simple lightcones. A special case is $\lambda$ real when factorisation does occur. 


\subsection{Example for Petrov Class I}

When the WLT is Petrov class I, there are four PNDs. A canonical form for the WLT in terms of the basis of the NP tetrad is

$$
C_{\mu \nu \sigma \tau}=\mu\left\{A_{\mu \nu} A_{\sigma \tau}+B_{\mu \nu} B_{\sigma \tau}\right\}+\lambda\left\{A_{\mu \nu} B_{\sigma \tau}+B_{\mu \nu} A_{\sigma \tau}+D_{\mu \nu} D_{\sigma \tau}\right\}+\text { c.c.. }
$$

We find that eq(30) takes the form eq(41) where the columns of $\mathcal{M}$ are

$$
\begin{aligned}
& \mathcal{M}_{1}=\left(\begin{array}{c}
q^{2}+(\lambda+\bar{\lambda})(l \cdot q n \cdot q+m \cdot q \bar{m} \cdot q) \\
-\mu(m \cdot q)^{2}-\bar{\mu}(\bar{m} \cdot q)^{2}-(\lambda+\bar{\lambda})(n \cdot q)^{2} \\
-\bar{\mu} l \cdot q \bar{m} \cdot q+(2 \lambda-\bar{\lambda}) n \cdot q m \cdot q \\
-\mu l \cdot q m \cdot q+(2 \bar{\lambda}-\lambda) n \cdot q \bar{m} \cdot q
\end{array}\right) \\
& \mathcal{M}_{2}=\left(\begin{array}{c}
-\mu(\bar{m} \cdot q)^{2}-\bar{\mu}(m . b)^{2}-(\lambda+\bar{\lambda})(l . q)^{2} \\
q^{2}+(\lambda+\bar{\lambda})(l \cdot q n . q+m . q \bar{m} . q) \\
-\mu n . q \bar{m} \cdot q+(2 \bar{\lambda}-\lambda) l . q m . q \\
-\bar{\mu} n . q m . q+(2 \lambda-\bar{\lambda}) l . q \bar{m} . q
\end{array}\right) \\
& \mathcal{M}_{3}=\left(\begin{array}{c}
\bar{\mu} n . q m \cdot q-(2 \lambda-\bar{\lambda}) l . q \bar{m} \cdot q \\
\mu l . q m \cdot q-(2 \bar{\lambda}-\lambda) n \cdot q \bar{m} \cdot q \\
q^{2}-(\lambda+\bar{\lambda})(l . q n \cdot q+m \cdot q \bar{m} \cdot q) \\
\mu(l . q)^{2}+\bar{\mu}(n \cdot q)^{2}+(\lambda+\bar{\lambda})(\bar{m} \cdot q)^{2}
\end{array}\right) \\
& \mathcal{M}_{4}=\left(\begin{array}{c}
\mu n \cdot q \bar{m} \cdot q-(2 \bar{\lambda}-\lambda) l \cdot q m \cdot q \\
\bar{\mu} l . q \bar{m} \cdot q-(2 \lambda-\bar{\lambda}) n \cdot q m \cdot q \\
\mu(n . q)^{2}+\bar{\mu}(l \cdot q)^{2}+(\lambda+\bar{\lambda})(m \cdot q)^{2} \\
q^{2}-(\lambda+\bar{\lambda})(l \cdot q n \cdot q+m \cdot q \bar{m} \cdot q)
\end{array}\right)
\end{aligned}
$$

The determinant of $\mathcal{M}$ is

$$
\operatorname{det} \mathcal{M}=\left(q^{2}\right)^{2}\left\{\Delta_{0}+\mu \Delta_{1}+\bar{\mu} \Delta_{2}+\mu^{2} \Delta_{3}+\bar{\mu}^{2} \Delta_{4}+\mu \bar{\mu} \Delta_{5}+\mu^{2} \bar{\mu} \Delta_{6}+\mu \bar{\mu}^{2} \Delta_{7}\right\},
$$

where

$$
\begin{aligned}
& \Delta_{0}=\frac{1}{4}(\lambda+\bar{\lambda}+1)(\lambda+\bar{\lambda}-2)\left(q^{2}\right)^{2}+18 \lambda \bar{\lambda} \text { l.qn.qm.qū. } . q, \\
& \Delta_{1}=-3 \bar{\lambda}(2 \lambda-\bar{\lambda}-2)\left[(l . q)^{2}(m . q)^{2}+(n . q)^{2}(\bar{m} . q)^{2}\right] \text {, } \\
& \Delta_{2}=-3 \lambda(2 \bar{\lambda}-\lambda-2)\left[(l . q)^{2}(\bar{m} . q)^{2}+(n . q)^{2}(m . q)^{2}\right] \text {, } \\
& \Delta_{3}=-\left[(\lambda+\bar{\lambda}+1)\left((l . q)^{2}(n . q)^{2}+(m . q)^{2}(\bar{m} . q)^{2}\right)+2(2 \bar{\lambda}-\lambda-1) l . q n . q m . q \bar{m} . q\right] \text {, }
\end{aligned}
$$

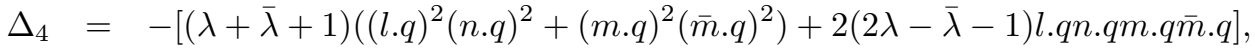

$$
\begin{aligned}
& \Delta_{5}=-(\lambda+\bar{\lambda}+1)\left[(l . q)^{4}+(n . q)^{4}+(m . q)^{4}+(\bar{m} . q)^{4}\right] \text {, } \\
& \Delta_{6}=-\left[(l . q)^{2}(\bar{m} . q)^{2}+(n . q)^{2}(m . q)^{2}\right], \\
& \Delta_{7}=-\left[(l . q)^{2}(m . q)^{2}+(n . q)^{2}(\bar{m} \cdot q)^{2}\right] .
\end{aligned}
$$

The quartic factor in eq(77) yields the dispersion relation for the physical modes. Unsurprisingly it does not exhibit any obvious factorisation properties. Hence we expect for this case also there are no simple lightcones controlling photon propagation. There may be special choices for the parameters that does allow factorisation.

\section{Generalised Gauge Fixing for the EM Field}

In the above discussion we obtained the dispersion relations for physical modes by imposing the gauge condition $g^{\mu \nu} \partial_{\mu} A_{\nu}(x)=0$. However in anticipation of issues that arise in the context of the renormalisation of gauge theories with a multi-metric structure we examine a more general 
form of gauge fixing for the electromagnetic (EM) field. We introduce a metric-like object $\Lambda^{\mu \nu}$ and impose the gauge condition

$$
\Lambda^{\mu \nu} \partial_{\mu} A_{\nu}(x)=0 .
$$

In appendix A we present the standard argument using the fuctional formalism to derive the gauge fixed action for the EM field. It is

$$
S_{(p)}=\int d^{n} x\left\{-\frac{1}{8} U^{\mu \nu \sigma \tau} F_{\mu \nu}(x) F_{\sigma \tau(x)}-\frac{1}{2} \Lambda^{\mu \nu} \Lambda^{\sigma \tau} \partial_{\mu} A_{\nu}(x) \partial_{\sigma} A_{\tau}(x)-\partial_{\mu} \bar{c}(x) \Lambda^{\mu \nu} \partial_{\nu} c(x)\right\} .
$$

Here $c(x)$ and $\bar{c}(x)$ are the ghost fields. Because we intend to use dimensional regularisation we express our results in $n$ dimensions. The argument identifying the preferred metric generalises straightforwardly to $n$ dimensions, hence we can express $U^{\mu \nu \sigma \tau}$ using eq(16) interpreted in $n$ dimensions.

The equation of motion for the photon field is then

$$
\left(g^{\mu \sigma} g^{\nu \tau}-\left(g^{\mu \tau} g^{\nu \sigma}-\Lambda^{\mu \nu} \Lambda^{\sigma \tau}\right)-C^{\mu \nu \sigma \tau}\right) \partial_{\sigma} \partial_{\mu} A_{\nu}(x)=0,
$$

and those for the ghost fields are

$$
\begin{aligned}
& \Lambda^{\mu \nu} \partial_{\mu} \partial_{\nu} c(x)=0 \\
& \Lambda^{\mu \nu} \partial_{\mu} \partial_{\nu} \bar{c}(x)=0 .
\end{aligned}
$$

Clearly $\Lambda^{\mu \nu}$ plays the role of the (inverse) metric for the ghost fields. It follows that the null mass-shell condition for the ghosts is

$$
\Lambda^{\mu \nu} q_{\mu} q_{\nu}=0 .
$$

Even in the case of no Lorentz symmetry breaking this null mass-shell is distinct from the photon null mass-shell unless we set $\Lambda^{\mu \nu}=g^{\mu \nu}$. In QED, of course the ghosts do not interact with the photons. For this reason the ghosts are usually ignored in QED calculations. We will keep them in mind in particular because they play a more significant role in the corresponding situation in non-abelian gauge theories. The presence of multiple null mass-shells or multiple lightcones in the theory raises the same same issues dealt with in a previous paper discussing a bimetric model with scalar fields. For the moment we will restrict our observations to the requirement that the parameters of the theory should be constrained so that there exist foliations of spacetime that are spacelike with respect to all relevant metrics in order to permit a causal structure in the theory including the ghosts [1].

\subsection{Photon Wavefunctions}

Plane wave solutions of eq(81) have the form

$$
A_{\nu}(x)=\varepsilon_{\nu} e^{-i q \cdot x},
$$

where $\varepsilon_{\nu}$ satisfies

$$
M^{\tau \nu} \varepsilon_{\nu}=0
$$

with

$$
M^{\tau \nu}=\mathcal{M}^{\tau \nu}-q^{\tau} q^{\nu}+Q^{\tau} Q^{\nu},
$$

where we have set $Q^{\mu}=\Lambda^{\mu \nu} q_{\nu}$ and where

$$
\mathcal{M}^{\tau \nu}=q^{2} g^{\tau \nu}-N^{\tau \nu} .
$$

Recall $N^{\tau \nu}=N^{\nu \tau}=C^{\mu \nu \sigma \tau} q_{\mu} q_{\sigma}$. In this notation the ghost mass shell condition is

$$
q \cdot Q=0
$$


We have also

$$
N^{\mu \nu} q_{\nu}=q_{\mu} N^{\mu \nu}=0 .
$$

We then find easily that eq(85) implies

$$
q \cdot Q Q \cdot \varepsilon=0 \text {. }
$$

Provided that $q$ does not lie on the ghost mass shell the photon wavefunction satisfies

$$
Q \cdot \varepsilon=0,
$$

which is precisely the gauge condition we wish to impose. In order that eq(85) have a solution it is necessary that $M^{\tau \nu}$ be singular. The inverse of $M^{\tau \nu}$ is $M_{\nu \lambda}$ satisfying $M^{\tau \nu} M_{\nu \lambda}=\delta_{\lambda}^{\tau}$ and has the form

$$
M_{\tau \nu}=\left(\delta_{\tau}^{\rho}-\frac{q_{\tau} Q^{\rho}}{Q \cdot q}\right) \mathcal{M}_{\rho \lambda}\left(\delta_{\nu}^{\lambda}-\frac{Q^{\lambda} q_{\nu}}{Q \cdot q}\right)+\frac{q_{\tau} q_{\nu}}{(Q \cdot q)^{2}},
$$

where $\mathcal{M}_{\rho \lambda}$ is the inverse of $\mathcal{M}^{\rho \lambda}$.

Clearly we can expect $M^{\tau \nu}$ to be singular when either

$$
Q^{\mu} q_{\mu}=\Lambda^{\mu \nu} q_{\mu} q_{\nu}=0
$$

or $\mathcal{M}^{\rho \lambda}$ is singular. That is either $q_{\mu}$ lies on the ghost mass-shell or $q_{\mu}$ satisfies $\operatorname{det} \mathcal{M}^{\rho \lambda}=0$. There may be special cases when these two constraints intersect and $q_{\mu}$ lies in the intersection. For simplicity of exposition we will not consider these special cases explicitly. The former condition above signals the presence of ghost contributions to the photon propagator. This is not different from the standard case except that our gauge condition separates the ghost massshell from that of the physical modes. The physical modes are associated with the vanishing of $\operatorname{det} \mathcal{M}^{\rho \lambda}$. However as we have seen from our examination of the various Petrov classes $\operatorname{det} \mathcal{M}^{\rho \lambda}$, in four dimensions, contains a factor $\left(q^{2}\right)^{2}$. This remains true in $n$ dimensions. Nevertheless $q^{2}=0$ does not correspond to a singularity of $M^{\nu \tau}$. We show this explicitly in appendix B.

The relationship between elements of the kernals of $M^{\tau \nu}$ and $\mathcal{M}^{\tau \nu}$ can be exhibited directly. Let $\varepsilon^{\prime}$ satisfy

$$
\mathcal{M}^{\tau \nu} \varepsilon_{\nu}^{\prime}=0
$$

It follows, assuming $q^{2} \neq 0$, that $q \cdot \varepsilon^{\prime}=0$. Now introduce $\varepsilon$ where

$$
\varepsilon=\varepsilon^{\prime}+\alpha q,
$$

$\alpha$ being chosen so that $Q \cdot \varepsilon=0$. It is then easy to check that

$$
M^{\tau \nu} \varepsilon_{\nu}=0 .
$$

This relationship, a gauge transformation of course, explains why the same physical dispersion relation emerges from either method of fixing the gauge. Because a factor of $\left(q^{2}\right)^{2}$ can be extracted from det $\mathcal{M}^{\tau \nu}$ it follows that the vanishing of the remaining factor is homogeneous in $q$ of degree $2(n-2)$ [11]. Confining attention to positive energy solutions we can expect in general $n-2$ branches for the physical disperion relation. As we have seen from our analysis of the Petrov classification, these may or may not factorise into separate lightlike mass-shells.

There is also a solution for which $\varepsilon \propto q$, when $q$ lies on the ghost mass-shell. The final contribution to the full suite of $n$ solutions is not pure plane wave but contains a secular term. It is not unique but can be chosen to have the form

$$
A_{\tau}(x)=\left(a_{\tau}+i q_{\tau} x^{0}\right) e^{-i q_{\mu} x^{\mu}},
$$

Here $q_{\tau}$ lies on the ghost mass-shell. The requirement that the above wavefunction is a solution of eq(81) only fixes $a_{\tau}$ up to a gauge transformation $a_{\tau} \rightarrow a_{\tau}+\alpha q_{\tau}$. It is convenient to complete the determination of $a_{\tau}$ by choosing $\alpha$ so that q.a $=0$. An analogous solution appears in conventional gauge fixing in standard gauge theories where it can be understood as the limit of a difference of two coinciding solutions in the Stuckelberg approach to gauge theories. 


\subsection{Photon Green's Functions}

We can compute the photon Green's functions from the generating functional, $Z_{(p)}[J]$, where

$$
Z_{(p)}[J]=\int d[A] \exp \left\{i S_{(p)}+i \int d^{n} x A_{\mu}(x) J^{\mu}(x)\right\}
$$

and

$$
S_{(p)}=\int d^{n} x\left\{-\frac{1}{8} U^{\mu \nu \sigma \tau} F_{\mu \nu}(x) F_{\sigma \tau}(x)-\frac{1}{2} \Lambda^{\mu \nu} \Lambda^{\sigma \tau} \partial_{\mu} A_{\nu}(x) \partial_{\sigma} A_{\tau}(x)\right\} .
$$

Here $S_{(p)}$ is the gauge fixed action without the ghosts which do not play a role in QED calculations.

On completing the square in the exponent in the functional integral we find that

$$
Z_{(p)}[J]=C \exp \left\{i \int d^{n} x d^{n} x^{\prime}\left(-\frac{1}{2} J^{\sigma}(x) N_{\sigma \tau}\left(x-x^{\prime}\right) J^{\tau}\left(x^{\prime}\right)\right)\right\}
$$

where

$$
N_{\tau \lambda}\left(x-x^{\prime}\right)=-\int \frac{d^{n} q}{(2 \pi)^{n}} e^{-i q_{\mu}\left(x-x^{\prime}\right)^{\mu}} M_{\tau \lambda}(q),
$$

The external factor $C=Z_{(p)}[0]$ and is irrelevant to subsequent calculations. It will be omitted from now on. The two-point Green's function for free photons is $i G_{F \mu \nu}\left(x-x^{\prime}\right)$ where

$$
i G_{F \mu \nu}\left(x-x^{\prime}\right)=\left.\frac{1}{Z[J]} \frac{\delta}{i \delta J^{\mu}(x)} \frac{\delta}{i \delta J^{\nu}(x)} Z[J]\right|_{J=0}=\int \frac{d^{n} q}{(2 \pi)^{n}} e^{-i q_{\mu}\left(x-x^{\prime}\right)^{\mu}}\left(-i M_{\mu \nu}(q)\right) .
$$

\subsection{Representation for the Photon Green's Function}

It is convenient, for later use in computing divergences, to construct a representation for the photon Green's function. The essential step is to obtain a representation for the core quantity $\mathcal{M}_{\rho \lambda}(q)$. First we introduce the matrix $\mathcal{M}_{\lambda}^{\mu}(q)$ given by

$$
\mathcal{M}_{\lambda}^{\mu}=\mathcal{M}^{\mu \nu}(q) g_{\nu \lambda}=q^{2} \delta_{\lambda}^{\mu}-N_{\lambda}^{\mu}(q),
$$

where

$$
N_{\lambda}^{\mu}(q)=N^{\mu \nu}(q) g_{\nu \lambda}=C_{\lambda}^{\mu \sigma}{ }^{\tau} q_{\sigma} q_{\tau} .
$$

Formally we write

$$
\mathcal{M}_{\lambda}^{\mu}(q)=(\mathcal{M}(q))_{\lambda}^{\mu},
$$

and

$$
N_{\lambda}^{\mu}(q)=(\mathbf{N}(q))_{\lambda}^{\mu},
$$

hence, in matrix notation,

$$
\mathcal{M}(q)=q^{2} 1-\mathbf{N}(q) .
$$

We have then

$$
\mathcal{M}_{\nu \rho}(q)=g_{\nu \lambda}\left(\mathcal{M}^{-1}(q)\right)_{\rho}^{\lambda}
$$

We now introduce the identity

$$
\mathcal{M}^{-1}(q)=-i \int_{0}^{\infty} d u \exp \{i u(\mathcal{M}(q)+i \varepsilon)\}=-i \int_{0}^{\infty} d u e^{i u\left(q^{2}+i \varepsilon\right)} \exp \{-i u \mathbf{N}(q)\} .
$$

We set

$$
\exp \{-i u \mathbf{N}(q)\}=\exp \left\{-i \mathbf{N}\left(-i \partial_{z}\right)\right\} e^{i \sqrt{u} q \cdot z},
$$

with the proviso that afterwards we set $z=0$. We then have the result

$$
\mathcal{M}^{-1}(q)=-i \exp \left\{-i \mathbf{N}\left(-i \partial_{z}\right)\right\} \int_{0}^{\infty} d u e^{i u\left(q^{2}+q \cdot z / \sqrt{u}+i \varepsilon\right)} .
$$

We will find this result useful later. It has the advantage of exhibiting the dependence of the photon propagator on the WLT, $C^{\mu \nu \sigma \tau}$. 


\subsection{Electron Green's Functions}

Because we wish to associate a new lightcone with the electron field we introduce a vierbein $\bar{e}^{a}{ }_{\mu}$ which allows us to create a new metric $\bar{g}_{\mu \nu}=\eta_{a b} \bar{e}^{a}{ }_{\mu} \bar{e}^{a}{ }_{\nu}$ and appropriate Dirac matrices $\bar{\gamma}^{\mu}=\bar{e}^{\mu}{ }_{a} \gamma^{a}$. Here $\gamma^{a}$ are standard Dirac matrices that satisfy $\left\{\gamma^{a}, \gamma^{b}\right\}=2 \eta^{a b}$, hence

$$
\left\{\bar{\gamma}^{\mu}, \bar{\gamma}^{\nu}\right\}=2 \bar{g}^{\mu \nu} .
$$

The volume element is again $d^{n} x$. This can be achieved by adjusting appropriately the normalisation of the electron field.

The action for the free electron field is $S_{(e)}$ where

$$
S_{(e)}=\int d^{n} x \bar{\psi}(x)\left(i \bar{\gamma}^{\mu} \partial_{\mu}-m\right) \psi(x),
$$

and $m$ is the mass parameter of the electron. The Green's functions for the elctron field are obtained from the generating functional $Z_{(e)}[\eta, \bar{\eta}]$ where

$$
Z_{(e)}[\eta, \bar{\eta}]=\int d[\psi] d[\bar{\psi}] \exp \left\{i S_{(e)}+i \int d^{n} x(\bar{\eta}(x) \psi(x)+\bar{\psi}(x) \eta(x))\right\},
$$

and $\eta(x)$ and $\bar{\eta}(x)$ are anticommuting fields. Completing the square in the exponent in the functional integral we find

$$
Z_{(e)}[\eta, \bar{\eta}]=C^{\prime} \exp \left\{-i \int d^{n} x d^{n} x^{\prime} \bar{\eta}(x) \Delta\left(x-x^{\prime}\right) \eta\left(x^{\prime}\right)\right\},
$$

where

$$
\Delta\left(x-x^{\prime}\right)=\int \frac{d^{n} q}{(2 \pi)^{n}} \frac{\left(\bar{\gamma}^{\mu} q_{\mu}+m\right) e^{-i q_{\mu}\left(x-x^{\prime}\right)^{\mu}}}{\bar{g}^{\mu \nu} q_{\mu} q_{\nu}-m^{2}+i \epsilon} .
$$

The coefficient $C^{\prime}$ can be omitted. We have

$$
i S_{F}\left(x-x^{\prime}\right)=\left.\frac{1}{Z[J, \eta, \bar{\eta}]} \frac{1}{i} \frac{\delta}{\delta \eta\left(x^{\prime}\right)} \frac{1}{i} \frac{\delta}{\delta \bar{\eta}(x)} Z[J, \eta, \bar{\eta}]\right|_{J=\eta=\bar{\eta}=0},
$$

giving the result

$$
i S_{F}\left(x-x^{\prime}\right)=i \int \frac{d^{n} q}{(2 \pi)^{n}} \frac{\left(\bar{\gamma}^{\mu} q_{\mu}+m\right) e^{-i q_{\mu}\left(x-x^{\prime}\right)^{\mu}}}{\bar{g}^{\mu \nu} q_{\mu} q_{\nu}-m^{2}+i \epsilon} .
$$

\section{Bimetric QED}

We now arrange for the electrons to interact with the electromagnetic field. Of course we must allow for the renormalisation of the bare parameters of the theory. We denote these bare parameters with a zero suffix. The bare electric charge is $-e_{0}$ and the bare mass parameter is $m_{0}$. From our previous paper we can anticipate that the metrics in the theory will also require renormalisation so we make the replacements $g_{\mu \nu} \rightarrow g_{0 \mu \nu}, \bar{e}_{\mu}^{a} \rightarrow \bar{e}_{0 \mu}^{a}$ and $\bar{\gamma}^{\mu} \rightarrow$ $\bar{\gamma}_{0}^{\mu}=\bar{e}_{0 a}^{\mu} \gamma^{a}$. We also have $\bar{g}_{\mu \nu} \rightarrow \bar{g}_{0 \mu \nu}=\eta_{a b} \bar{e}_{0 \mu}^{a} \bar{e}_{0 \nu}^{b}$. We must also allow for the necessity of renormalising the the WLT representing Lorentz symmetry breaking for the photon field and make the replacement $C^{\mu \nu \sigma \tau} \rightarrow C_{0}^{\mu \nu \sigma \tau}$ and hence the obvious corresponding replacement $U^{\mu \nu \sigma \tau} \rightarrow U_{0}^{\mu \nu \sigma \tau}$. In addition the gauge fixing metric is modified with $\Lambda^{\mu \nu} \rightarrow \Lambda_{0}^{\mu \nu}$, in order to accommodate possible renormalisation effects.

We deviate a little from the approach of [1] by identifying the volume elements of the two metrics as $d^{n} x$ which then remains unrenormalized. Instead we we rely in a conventional way, on field renormalisations to render the Green's functions of the theory finite. In [1] we absorbed this field renormalisation into the renormalisation of the volume elements. In fact a study of the renormalisation group in [1] showed that it may be factored out again. In the present model 
it turns out that we cannot in any case, carry out this manoeuvre because of the photon action depends quadratically on the (inverse) metric. We will therefore adopt the more conventional scheme and treat the field renormalisations independently of the metric.

The action for the electromagnetic field $A_{\mu}(x)$ is the gauge fixed action $S_{(p)}$ is now given by eq(99) with the replacement of parameters by their bare versions. We omit the ghost fields from now on since they play no role in QED calculations. The action for the electron field is $S_{(e)}$ is obtained similarly from eq(113) together with the inclusion of the elctromagnetic interaction to yield

$$
S_{(e)}=\int d^{n} x \bar{\psi}(x)\left(i \bar{\gamma}_{0}^{\mu} \partial_{\mu}+e_{0} \bar{\gamma}_{0}^{\mu} A_{\mu}(x)-m_{0}\right) \psi(x)
$$

The total action for the theory is

$$
S=S_{(p)}+S_{(e)}
$$

The generating functional for the Green's functions is

$$
Z[J, \eta, \bar{\eta}]=\int d[A] d[\psi] d[\bar{\psi}] \exp \left\{i S+i \int d^{n} x\left(J^{\mu}(x) A_{\mu}(x)+(\bar{\eta}(x) \psi(x)+\bar{\psi}(x) \eta(x))\right)\right\}
$$

When $e_{0}=0 S$ reduces to $S_{f}$ the free action and $Z[J, \eta, \bar{\eta}]$ becomes the free-particle generating functional, $Z_{f}[J, \eta, \bar{\eta}]$, where

$$
Z_{f}[J, \eta, \bar{\eta}]=\int d[A] d[\psi] d[\bar{\psi}] \exp \left\{i S_{f}+i \int d^{n} x\left(J^{\mu}(x) A_{\mu}(x)+(\bar{\eta}(x) \psi(x)+\bar{\psi}(x) \eta(x))\right)\right\}
$$

The full generating functional, $Z[J, \eta, \bar{\eta}]$, can be reconstructed from $Z_{f}[J, \eta, \bar{\eta}]$ by means of the standard formula

$$
Z[J, \eta, \bar{\eta}]=\exp \left\{i e_{0} \int d^{n} x \frac{1}{i} \frac{\delta}{\delta \eta(x)} \bar{\gamma}_{0}^{\mu} \frac{1}{i} \frac{\delta}{\delta J^{\mu}(x)} \frac{1}{i} \frac{\delta}{\delta \bar{\eta}(x)}\right\} Z_{f}[J, \eta, \bar{\eta}] .
$$

\subsection{Green's Functions with Interaction}

The Green's functions that are important for a study of the renormalisation of the theory are the two point Green's function for photons, $G_{\mu \nu}\left(x-x^{\prime}\right)$, where

$$
i G_{\mu \nu}\left(x-x^{\prime}\right)=\left.\frac{1}{Z[J, \eta, \bar{\eta}]} \frac{1}{i} \frac{\delta}{\delta J^{\mu}(x)} \frac{1}{i} \frac{\delta}{\delta J^{\nu}\left(x^{\prime}\right)} Z[J, \eta, \bar{\eta}]\right|_{J=\eta=\bar{\eta}=0}
$$

the two point Green's function for electrons, $S\left(x-x^{\prime}\right)$, where

$$
i S\left(x-x^{\prime}\right)=\left.\frac{1}{Z[J, \eta, \bar{\eta}]} \frac{1}{i} \frac{\delta}{\delta \eta\left(x^{\prime}\right)} \frac{1}{i} \frac{\delta}{\delta \bar{\eta}(x)} Z[J, \eta, \bar{\eta}]\right|_{J=\eta=\bar{\eta}=0} .
$$

and the vertex function $V_{\mu}\left(x, x^{\prime}, y\right)$, where

$$
i V_{\mu}\left(x, x^{\prime}, y\right)=\left.\frac{1}{Z[J, \eta, \bar{\eta}]} \frac{1}{i} \frac{\delta}{J^{\mu}(y)} \frac{1}{i} \frac{\delta}{\delta \eta\left(x^{\prime}\right)} \frac{1}{i} \frac{\delta}{\delta \bar{\eta}(x)} Z[J, \eta, \bar{\eta}]\right|_{J=\eta=\bar{\eta}=0} \cdot
$$

The lowest approximation to $G_{\mu \nu}\left(x-x^{\prime}\right)$ is obtained by substituting $Z_{f}$ for $Z$ in eq(124). We have

$$
i G_{F \mu \nu}\left(x-x^{\prime}\right)=\frac{1}{i} \int \frac{d^{n} q}{(2 \pi)^{n}} e^{-i q_{\mu}\left(x-x^{\prime}\right)^{\mu}} M_{0 \mu \nu}(q) .
$$

We have made the substitution $M_{\mu \nu} \rightarrow M_{0 \mu \nu}$ to indicate that bare parameters are involved in the construction of $M_{0 \mu \nu}$.

In the same way we obtain the lowest approximation to $S\left(x-x^{\prime}\right)$. It is

$$
i S_{F}\left(x-x^{\prime}\right)=-\frac{1}{i} \int \frac{d^{n} q}{(2 \pi)^{n}} \frac{\left(\bar{\gamma}_{0}^{\mu} q_{\mu}+m_{0}\right) e^{-i q_{\mu}\left(x-x^{\prime}\right)^{\mu}}}{\bar{g}_{0}^{\mu \nu} q_{\mu} q_{\nu}-m_{0}^{2}+i \epsilon} .
$$

The lowest approximation to $i V_{\mu}\left(x, x^{\prime}, y\right)$ is

$$
i V_{F \mu}\left(x, x^{\prime}, y\right)=\int d^{n} y^{\prime} i G_{F \mu \nu}\left(y-y^{\prime}\right) i S_{F}\left(x-y^{\prime}\right)\left(i e_{0} \bar{\gamma}_{0}^{\nu}\right) i S_{F}\left(y^{\prime}-x^{\prime}\right) .
$$




\subsection{Feynman Rules for Bimetric QED}

The Feynman rules for computing the perturbative expansions of Green's functions (in terms of bare parameters) can be read off from the expansion for $Z[J, \eta, \bar{\eta}]$ in eq(123) and the Green's function formulae in eqs(124), (125). The Feynman diagrams are the conventional ones with the lines corresponding to $i G_{F \mu \nu}\left(x-x^{\prime}\right)$ for photons, $i S_{F}\left(x-x^{\prime}\right)$ for electrons and $i e_{0} \bar{\gamma}_{0 \mu}$ for each vertex. In momentum space a photon line with momentum $q_{\mu}$ is associated with a factor

$$
i G_{F \mu \nu}(q)=-i M_{0 \mu \nu}(q)
$$

and each electron line with

$$
i S_{F}(q)=i \frac{\left(\bar{\gamma}_{0}^{\mu} q_{\mu}+m_{0}\right)}{\bar{g}_{0}^{\mu \nu} q_{\mu} q_{\nu}-m_{0}^{2}+i \epsilon} .
$$

The vertex is $i e_{0} \bar{\gamma}_{0 \mu}$. Of course there is momentum conservation at each vertex and each loop momentum $q_{\mu}$ is integrated with a measure

$$
\int \frac{d^{n} q}{(2 \pi)^{n}}
$$

Each electron loop has associated with it a factor of $(-1)$.

\subsection{Renormalisation}

We use dimensional regularisation with minimal subtraction to renormalise the theory. We introduce a scale $\mu$ so that the electron charge can be expressed in the form

$$
e_{0}=(\mu)^{(4-n) / 2} e\left(1+\sum_{k=1}^{\infty} a^{(k)}(n)\left(e^{2}\right)^{k}\right) .
$$

The term in the sum that is $O\left(e^{2}\right)$ is simply a single pole at $n=4$. The renormalized charge $e$ is dimensionless. Similarly the bare mass is expressed in the form

$$
m_{0}=m\left(1+\sum_{k=1}^{\infty} b^{(k)}(n)\left(e^{2}\right)^{k}\right),
$$

where $m$ is the renormalized mass parameter. Again the term that is $O\left(e^{2}\right)$ is a simple pole at $n=4$.

The new aspect of the renormalisation procedure required for bimetric QED is that we must also allow for a renormalisation of the metrics thus

$$
g_{0}^{\mu \nu}=g^{\mu \nu}+\sum_{k=1}^{\infty} g^{(k) \mu \nu}\left(e^{2}\right)^{k} .
$$

Similarly for the vierbein associated with the electron

$$
\bar{e}_{0 a}^{\mu}=\bar{e}_{a}^{\mu}+\sum_{k=1}^{\infty} \bar{e}_{a}^{(k) \mu}\left(e^{2}\right)^{k} .
$$

The WLT term also requires renormalisation

$$
C_{0}^{\mu \nu \sigma \tau}=C^{\mu \nu \sigma \tau}+\sum_{k=1}^{\infty} C^{(k) \mu \nu \sigma \tau}\left(e^{2}\right)^{k} .
$$

Finally

$$
\Lambda_{0}^{\mu \nu}=\Lambda^{\mu \nu}+\sum_{k=1}^{\infty} \Lambda^{(k) \mu \nu}\left(e^{2}\right)^{k} .
$$




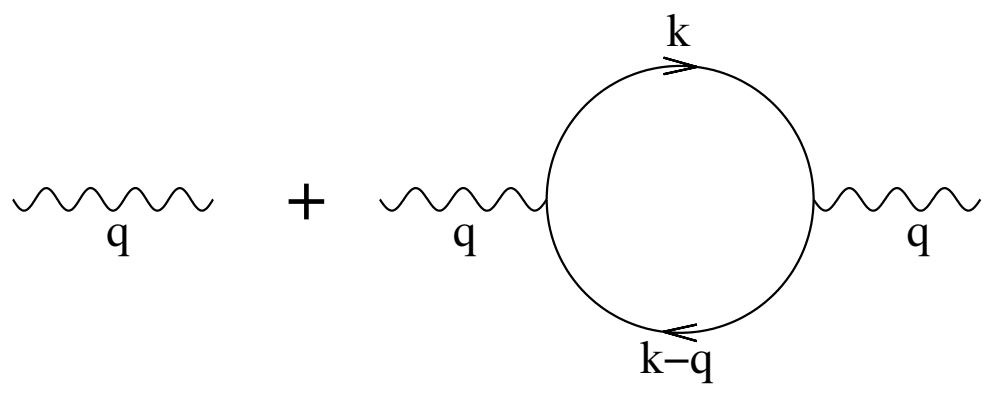

Figure 1: Contributions to the photon propagator to $O\left(e_{0}^{2}\right)$.

Again the terms of $O\left(e^{2}\right)$ contain a simple pole at $n=4$. Higher order terms contain poles of increasingly higher order. Note that since we require $\operatorname{det} g_{0}^{\mu \nu}=\operatorname{det} g^{\mu \nu}=-1$, it follows that

$$
g_{\mu \nu} g^{(1) \mu \nu}=0 .
$$

The renormalisation procedure is carried out by inserting these parameter expansions into the bare perturbation series and re-expanding in the renormalised charge $e$. The $n$-dependent coefficients of powers of $e^{2}$ are adjusted so that there are sufficient cancellations of poles at $n=4$ that residual singular structure can be removed by appropriate field renormalisation factors yielding finally Green's functions that are without divergent terms at $n=4$.

\section{$7 \quad$ Vacuum Polarisation}

The lowest order contribution to the photon two-point function is represented by the Feynman diagram in Fig 1.

Making use of the Feynman rules above we obtain to order $e_{0}^{2}$

$$
i G_{\mu \nu}(q)=i G_{F \mu \nu}(q)+i G_{F \mu \sigma}(q)\left[i \Sigma^{\sigma \tau}(q)\right] i G_{F \tau \nu}(q),
$$

where

$$
i \Sigma^{\sigma \tau}(q)=(-1)\left(i e_{0}\right)^{2} \int \frac{d^{n} k}{(2 \pi)^{n}} \operatorname{Tr}\left\{\bar{\gamma}_{0}^{\sigma} i S_{F}(k-q) \bar{\gamma}_{0}^{\tau} i S_{F}(k) \bar{\gamma}_{0}^{\tau}\right\} .
$$

If we introduce the inverse of the Green's function, $G^{\mu \nu}(q)$ where $G^{\mu \nu}(q) G_{\nu \lambda}(q)=\delta_{\lambda}^{\mu}$ and correspondingly $G_{F}^{\mu \nu}(q)$ where $G_{F}^{\mu \nu}(q) G_{F \nu \lambda}(q)=\delta_{\lambda}^{\mu}$ we find to second order

$$
G^{\mu \nu}(q)=G_{F}^{\mu \nu}(q)+\Sigma^{\mu \nu}(q) .
$$

Since our calculation is an expansion to one loop we restrict the coupling constant expansion to $O\left(e^{2}\right)$. We have then

$$
\Sigma^{\mu \nu}(q)=i e^{2} \mu^{(4-n)} \int \frac{d^{n} k}{(2 \pi)^{n}} \frac{\operatorname{Tr}\left[\bar{\gamma}^{\mu}\left(\bar{\gamma}^{\lambda}(k-q)_{\lambda}+m\right) \bar{\gamma}^{\nu}\left(\bar{\gamma}^{\rho} k_{\rho}+m\right)\right]}{\left(\bar{g}^{\alpha \beta}(k-q)_{\alpha}(k-q)_{\beta}-m^{2}+i \epsilon\right)\left(\bar{g}^{\mu \nu} k_{\mu} k_{\nu}-m^{2}+i \epsilon\right)} .
$$

The calculation can be performed along essentially conventional lines. The divergent behaviour is exhibited by calculating to $O\left(q^{2}\right)$ and we find

$$
\Sigma^{\mu \nu}(q) \simeq-\frac{e^{2}}{3} \frac{d(n)}{(4 \pi)^{n / 2}}\left(\frac{\mu}{m}\right)^{4-n} \Gamma(2-n / 2)\left(\bar{g}^{\mu \nu} \bar{g}^{\alpha \beta}-\bar{g}^{\mu \beta} \bar{g}^{\alpha \nu}\right) q_{\alpha} q_{\beta} .
$$

Here $d(n)$ is the dimension of the $\gamma$-matrix representation. Of course $d(4)=4$. Therefore we have a pole at $n=4$ of the form

$$
\Sigma^{\mu \nu}(q) \simeq \frac{e^{2}}{6 \pi^{2}} \frac{1}{n-4}\left(\bar{g}^{\mu \nu} \bar{g}^{\alpha \beta}-\bar{g}^{\mu \beta} \bar{g}^{\alpha \nu}\right) q_{\alpha} q_{\beta} .
$$


Let the tensor $W^{\mu \alpha \nu \beta}$ be given by

$$
W^{\mu \alpha \nu \beta}=\bar{g}^{\mu \nu} \bar{g}^{\alpha \beta}-\bar{g}^{\mu \beta} \bar{g}^{\alpha \nu} .
$$

Since it has the same symmetry properties as $U^{\mu \alpha \nu \beta}, W^{\mu \alpha \nu \beta}$ can (in four dimensions) be expressed in the form

$$
W^{\mu \alpha \nu \beta}=\frac{1}{12} W\left(g^{\mu \nu} g^{\alpha \beta}-g^{\mu \beta} g^{\alpha \nu}\right)+\frac{1}{2}\left(V^{\mu \nu} g^{\alpha \beta}+g^{\mu \nu} V^{\alpha \beta}-V^{\mu \beta} g^{\alpha \nu}-g^{\mu \beta} V^{\alpha \nu}\right)-V^{\mu \nu \sigma \tau},
$$

where

$$
\begin{aligned}
W^{\mu \nu} & =W^{\mu \alpha \nu \beta} g_{\alpha \beta}, \\
W & =W^{\mu \nu} g_{\mu \nu}, \\
V^{\mu \nu} & =W^{\mu \nu}-\frac{1}{4} W g^{\mu \nu}, \\
V^{\mu \alpha \sigma \beta} g_{\alpha \beta} & =0 .
\end{aligned}
$$

Here $V^{\mu \alpha \nu \beta}$ is a WLT constructed ultimately from $\bar{g}^{\mu \nu}$. Later we will examine how different choices for $\bar{g}^{\mu \nu}$ influence the Petrov class of $V^{\mu \alpha \nu \beta}$. Of course there is an $n$-dimensional version of this argument. However since we are simply calculating pole resiues at $n=4$ we will find here and later that the 4-dimensional calculation is sufficient.

From eq(127) and eq(139) we see that

$$
G_{F}^{\mu \nu}(q)=-M_{0}^{\mu \nu}(q)+\Sigma^{\mu \nu}(q)
$$

that is

$$
G_{F}^{\mu \nu}(q)=-\left(g_{0}^{\mu \nu} g_{0}^{\alpha \beta}-g_{0}^{\mu \beta} g_{0}^{\alpha \nu}+\Lambda_{0}^{\mu \beta} \Lambda_{0}^{\alpha \nu}-C_{0}^{\mu \alpha \nu \beta}-\frac{e^{2}}{6 \pi^{2}} \frac{1}{n-4} W^{\mu \alpha \nu \beta}\right) q_{\alpha} q_{\beta}
$$

Using the expansions to $O\left(e^{2}\right)$ in eq(132) to eq(134) we see that we can remove some of the UV poles at $n=4$ by choosing

$$
e^{2} g^{(1) \mu \nu}=\frac{e^{2}}{12 \pi^{2}} \frac{1}{n-4} V^{\mu \nu}
$$

and

$$
e^{2} C^{(1) \mu \alpha \nu \beta}=\frac{e^{2}}{6 \pi^{2}} \frac{1}{n-4} V^{\mu \alpha \nu \beta}-\frac{e^{2}}{72 \pi^{2}} \frac{W}{n-4} C^{\mu \alpha \nu \beta},
$$

with the result

$$
G_{F}^{\mu \nu}(q)=-\left\{\left(1-\frac{e^{2}}{72 \pi^{2}} \frac{W}{n-4}\right)\left(g^{\mu \nu} g^{\alpha \beta}-g^{\mu \beta} g^{\alpha \nu}-C^{\mu \alpha \nu \beta}\right)+\Lambda_{0}^{\mu \beta} \Lambda_{0}^{\alpha \nu}\right\} q_{\alpha} q_{\beta} .
$$

This may be expressed in the form

$$
G_{F}^{\mu \nu}(q)=-\left(1-\frac{e^{2}}{72 \pi^{2}} \frac{W}{n-4}\right)\left\{\left(g^{\mu \nu} g^{\alpha \beta}-g^{\mu \beta} g^{\alpha \nu}\right)+\Lambda^{\mu \beta} \Lambda^{\alpha \nu}\right\} q_{\alpha} q_{\beta},
$$

provided we arrange the expansion for the ghost mass-shell metric to satisfy

$$
\Lambda_{0}^{\mu \beta}=\left(1-\frac{e^{2}}{144 \pi^{2}} \frac{W}{n-4}\right) \Lambda^{\mu \beta} .
$$

The renormalised Green's function, $G_{R F}^{\mu \nu}$ is then obtained by means of the appropriate multiplicative photon wavefunction renormalisation yielding

$$
G_{R F}^{\mu \nu}(q)=-\left\{\left(g^{\mu \nu} g^{\alpha \beta}-g^{\mu \beta} g^{\alpha \nu}\right)+\Lambda^{\mu \beta} \Lambda^{\alpha \nu}-C^{\mu \alpha \nu \beta}\right\} q_{\alpha} q_{\beta} .
$$

The reason then that we introduced a distinct ghost mass-shell metric was to permit this multiplicative renormalisation for the photon Green's function. 


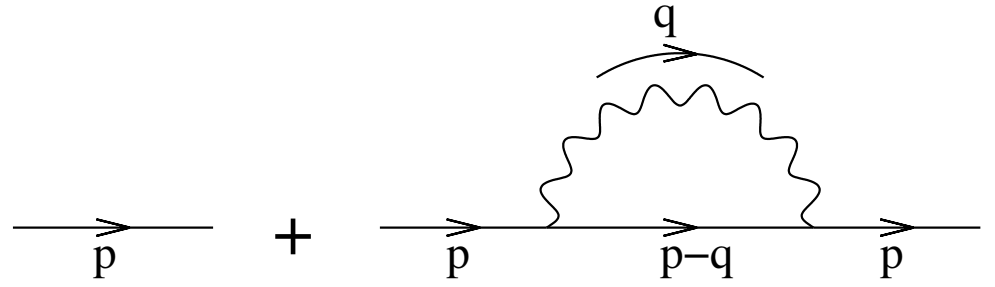

Figure 2: Contributions to the electron propagator to $O\left(e_{0}^{2}\right)$.

\section{Electron Propagator}

The lowest contributions to the electron propagator are shown in Fig 2.

To $O\left(e_{0}^{2}\right)$ we have

$$
i S(p)=i S_{F}(p)+i S_{F}(p) i \Sigma(p) i S_{F}(p),
$$

where

$$
i \Sigma(p)=\left(i e_{0}\right)^{2} \int \frac{d^{n} q}{(2 \pi)^{n}} \bar{\gamma}_{0}^{\mu} i S_{F}(p-q) \bar{\gamma}_{0}^{\nu}\left(-i M_{0 \mu \nu}(q)\right) .
$$

To second order we have

$$
S^{-1}(p)=S_{F}^{-1}(p)+\Sigma(p) .
$$

Restricting the calculation to $O\left(e^{2}\right)$ we find

$$
\Sigma(p)=(i e)^{2} \mu^{4-n} \int \frac{d^{n} q}{(2 \pi)^{n}} \bar{\gamma}^{\mu} \frac{\left(\bar{\gamma}^{\sigma}\left(p_{\sigma}-q_{\sigma}\right)+m\right)}{\bar{g}^{\alpha \beta}\left(p_{\alpha}-q_{a}\right)\left(p_{\beta}-q_{\beta}\right)-m^{2}+i \varepsilon} \bar{\gamma}^{\nu}\left(-i M_{\mu \nu}(q)\right) .
$$

The UV divergences in $\Sigma(p)$ are contained in the first two twrms of the Taylor series

$$
\Sigma(p)=\Sigma(0)+p_{\mu} \Sigma^{\mu}(0)+O\left(p^{2}\right),
$$

where

$$
\Sigma^{\mu}(0)=\left.\frac{\partial}{\partial p_{\mu}} \Sigma(p)\right|_{p=0}
$$

We have then

$$
\Sigma(0)=(i e)^{2} \mu^{4-n} m \int \frac{d^{n} q}{(2 \pi)^{n}} \frac{\bar{g}^{\mu \nu}}{\bar{g}^{\alpha \beta} q_{\alpha} q_{\beta}-m^{2}+i \varepsilon}\left(-i M_{\mu \nu}(q)\right),
$$

and

$$
\Sigma^{\tau}(0)=-(i e)^{2} \mu^{4-n} \int \frac{d^{n} q}{(2 \pi)^{n}} \frac{\bar{\gamma}^{\mu}\left(\bar{\gamma}^{\sigma} q_{\sigma}-m\right) \bar{\gamma}^{\tau}\left(\bar{\gamma}^{\rho} q_{\rho}-m\right) \bar{\gamma}^{\nu}}{\left(\bar{\gamma}^{\alpha \beta} q_{\alpha} q_{\beta}-m^{2}\right)^{2}}\left(-i M_{\mu \nu}(q)\right) .
$$

It follows that

$$
\Sigma^{\tau}(0)=H_{\rho}^{\tau} \bar{\gamma}^{\rho}
$$

where

$$
H_{\rho}^{\tau}=H_{\rho}^{(1) \tau}+H_{\rho}^{(2) \tau},
$$

with

$$
H_{\rho}^{(1) \tau}=(i e)^{2} \mu^{4-n} \int \frac{d^{n} q}{(2 \pi)^{n}} \frac{\delta_{\rho}^{\mu} \bar{g}^{\tau \nu}+\delta_{\rho}^{\nu} \bar{g}^{\tau \mu}-\delta_{\rho}^{\tau} \bar{g}^{\mu \nu}}{\bar{g}^{\alpha \beta} q_{\alpha} q_{\beta}-m^{2}+i \varepsilon}\left(-i M_{\mu \nu}(q)\right),
$$

and

$$
H_{\rho}^{(2) \tau}=-2(i e)^{2} \mu^{4-n} \int \frac{d^{n} q}{(2 \pi)^{n}} \frac{\bar{g}^{\tau \beta} q_{\beta} q_{\sigma}\left(\delta_{\rho}^{\mu} \bar{g}^{\sigma \nu}+\delta_{\rho}^{\nu} \bar{g}^{\sigma \mu}-\delta_{\rho}^{\sigma} \bar{g}^{\mu \nu}\right)}{\left(\bar{g}^{\alpha \beta} q_{\alpha} q_{\beta}-m^{2}+i \varepsilon\right)^{2}}\left(-i M_{\mu \nu}(q)\right) .
$$



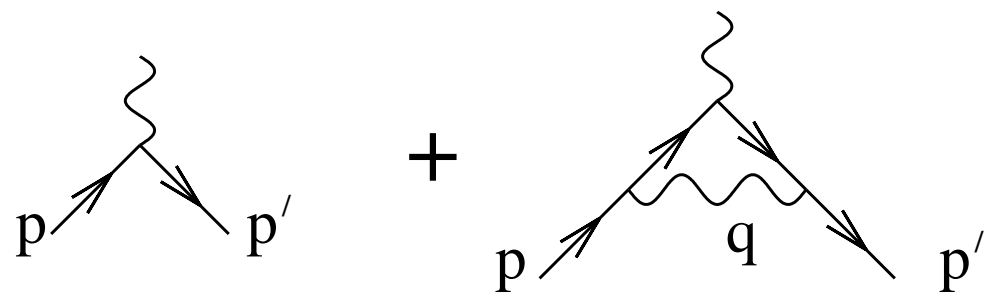

Figure 3: Contributions to the electron-photon vertex to $O\left(e_{0}^{3}\right)$.

It is useful to split $H_{\rho}^{\tau}$ into a trace part and a traceles part,

$$
H_{\rho}^{\tau}=\frac{1}{n} H \delta_{\rho}^{\tau}+h_{\rho}^{\tau},
$$

where

$$
H=H_{\tau}^{\tau},
$$

and

$$
h_{\tau}^{\tau}=0 .
$$

The pole at $n=4$ in $H$ determines the field renormalisation of the electron propagator while the pole in $h_{\rho}^{\tau}$ fixes the counter term in $e_{0 a}^{\mu}$. We have then from eq(156)

$$
S^{-1}(p)=\bar{\gamma}_{0}^{\mu} p_{\mu}-m_{0}+\Sigma(0)+\Sigma^{\mu}(0) p_{\mu},
$$

where we retain only the pole contributions in $\Sigma(0)$ etc. Using eq(131) and eq(133) we have

$$
S^{-1}(p)=\left(\bar{e}_{a}^{\mu}+e^{2} \bar{e}_{a}^{(1) \mu}\right) \gamma^{a} p_{\mu}-m\left(1+e^{2} b^{(1)}\right)+\Sigma(0)+\left(h_{\rho}^{\mu}+\frac{1}{4} H \delta_{\rho}^{\mu}\right) \bar{\gamma}^{\rho} p_{\mu} .
$$

If we set

$$
e^{2} \bar{e}_{a}^{(1) \mu} \bar{e}_{\rho}^{a}=-h_{\rho}^{\mu}
$$

and

$$
m e^{2} b^{(1)}=\frac{1}{4} m H+\Sigma(0)
$$

then eq(156) becomes

$$
S^{-1}(p)=\left(1+\frac{1}{4} H\right)\left(\bar{\gamma}^{\mu} p_{\mu}-m\right) .
$$

Finally we see that the field renormalisation for the electron is

$$
Z_{e}=\left(1-\frac{1}{4} H\right)
$$

Hence the renormalized inverse propagator $S_{R}^{-1}(p)=Z_{e} S^{-1}(p)$ is finite to $O\left(e^{2}\right)$. It is useful to note that eq(171) implies

$$
\bar{g}_{0}^{\mu \nu}=\bar{g}^{\mu \nu}-h_{\rho}^{\mu} \bar{g}^{\rho \nu}-h_{\rho}^{\nu} \bar{g}^{\rho \mu} .
$$

\section{$9 \quad$ Vertex}

The complete vertex amplitude to $O\left(e^{3}\right)$ corresponds to the diagrams in Fig 3 . It has the form $V^{\mu}\left(p, p^{\prime}\right)=i e_{0} \bar{\gamma}^{\tau}+\mathcal{V}^{\tau}\left(p, p^{\prime}\right)$ where

$$
\mathcal{V}^{\tau}\left(p, p^{\prime}\right)=(i e)^{3} \int \frac{d^{n} q}{(2 \pi)^{n}} \frac{\bar{\gamma}^{\mu} i\left(\bar{\gamma}^{\sigma}\left(p_{\sigma}^{\prime}-q_{\sigma}\right)+m\right) \bar{\gamma}^{\tau} i\left(\bar{\gamma}^{\rho}\left(p_{\rho}-q_{\rho}\right)+m\right) \bar{\gamma}^{\nu}\left(-i M_{\mu \nu}(q)\right)}{\left(\bar{g}^{\alpha^{\prime} \beta^{\prime}}\left(p_{\alpha^{\prime}}^{\prime}-q_{\alpha^{\prime}}\right)\left(p_{\beta^{\prime}}^{\prime}-q_{\beta^{\prime}}\right)-m^{2}\right)\left(\bar{g}^{\alpha \beta}\left(p_{\alpha}-q_{\alpha}\right)\left(p_{\beta}-q_{\beta}\right)-m^{2}\right)}
$$


The divergence is contained in

$$
\mathcal{V}^{\tau}(0,0)=-(i e)^{3} \int \frac{d^{n} q}{(2 \pi)^{n}} \bar{\gamma}^{\mu} \frac{\left(\bar{g}^{\sigma} q_{\sigma}-m\right) \bar{\gamma}^{\tau}\left(\bar{\gamma}^{\rho} q_{\rho}-m\right)}{\left(\bar{\gamma}^{\alpha \beta} q_{\alpha} q_{\beta}-m^{2}\right)^{2}} \bar{\gamma}^{\nu}\left(-i M_{\mu \nu}(q)\right) .
$$

It follows that

$$
\mathcal{V}^{\tau}(0,0)=i e \Sigma^{\tau}(0) .
$$

Hence the divergence of $\mathcal{V}^{\tau}(0,0)$ is the same as that of $\Sigma^{\tau}(0)$ up to a factor $i e$. Using eq(130) we have

$$
V^{\mu}(0,0)=\mu^{4-n} i e\left(1+e^{2} a^{(1)}\right)\left(\bar{e}_{a}^{\mu}+e^{2} \bar{e}_{a}^{(1) \mu}\right) \gamma^{a}+i e \Sigma^{\mu}(0) .
$$

It follows that if we use eq(171) we find to $O\left(e^{3}\right)$

$$
V^{\mu}(0,0)=i e\left(\left(1+\frac{1}{4} H+e^{2} a^{(1)}\right) \bar{\gamma}^{\mu}=i e\left(1+\frac{1}{4} H\right)\left(1+e^{2} a^{(1)}\right) \bar{\gamma}^{\mu} .\right.
$$

The renormalized vertex $V_{R}^{\mu}(0,0)=Z_{e} \sqrt{Z_{\gamma}} V^{\mu}(0,0)$ is finite provided we set

$$
1+e^{2} a^{(1)}=\frac{1}{\sqrt{Z_{\gamma}}}
$$

that is

or

$$
e^{2} a^{(1)}=-\frac{e^{2}}{144 \pi^{2}} \frac{W}{n-4},
$$

$$
e_{0}^{2}=\mu^{4-n} e^{2}\left(1-\frac{e^{2}}{72 \pi^{2}} \frac{W}{n-4}\right)
$$

\section{Pole Divergences at $n=4$}

The pole divergences at $n=4$ can be exhibited explicitly by making use of the photon propagator representation in eq(111). For example from eq(160) we find

$$
\Sigma(0)=-i(i e)^{2} \mu^{4-n} m \bar{g}^{\mu \nu} g_{\mu \rho} \exp \left\{-i \mathbf{N}\left(\partial_{z}\right)\right\}^{\rho}{ }_{\nu} L(z),
$$

where

$$
L(z)=-i \int d u \int \frac{d^{n} q}{(2 \pi)^{n}} \frac{1}{\bar{g}^{\alpha \beta} q_{\alpha} q_{\beta}-m^{2}+i \varepsilon} e^{i u\left(q^{2}+z \cdot q / \sqrt{u}+i \varepsilon\right)},
$$

and where finally we set $z=0$. We can now express $L(z)$ in the form

$$
\begin{aligned}
L(z) & =(-i)^{2} \int d u d v \int \frac{d^{n} q}{(2 \pi)^{n}} e^{i u\left(q^{2}+z \cdot q / \sqrt{u}\right)+i v\left(\bar{g}^{\alpha \beta} q_{\alpha} q_{\beta}-m^{2}+i \varepsilon\right)} \\
& =(-i)^{2} \int_{0}^{1} d x \int_{0}^{\infty} d \lambda \lambda \int \frac{d^{n} q}{(2 \pi)^{n}} e^{i \lambda\left(\hat{g}^{\alpha \beta}(x) q_{\alpha} q_{\beta}+x z^{\alpha} q_{\alpha} / \sqrt{u}-m^{2}(1-x)+i \varepsilon\right)},
\end{aligned}
$$

where we have set $u=\lambda x$ and $v=\lambda(1-x)$ and have introduced the interpolated (inverse) metric $\hat{g}^{\alpha \beta}(x)=x g^{\alpha \beta}+(1-x) \bar{g}^{\alpha \beta}$. This metric was introduced in reference [1] where it was emphasised that it should remain non-singular for $0 \leq x \leq 1$ as a condition of acceptable causal structure. This was ensured by the requirement that lightcones associated with $g_{\alpha \beta}$ and $\bar{g}_{\alpha \beta}$ overlap so that there exists a shared set of spacetime vectors that are timelike in both metrics. The same point holds here. Making this assumption we can evaluate $L(z)$ as

$$
L(z)=(-i)^{2} \int_{0}^{1} d x \int_{0}^{\infty} d \lambda \lambda \int \frac{d^{n} q^{\prime}}{(2 \pi)^{n}} e^{i \lambda\left(\hat{g}^{\alpha \beta} q_{\alpha}^{\prime} q_{\beta}^{\prime}+x^{2} \hat{g}_{\alpha \beta} z^{\alpha} z^{\beta} /(4 u)-m^{2}(1-x)+i \varepsilon\right)}
$$


where $q_{\alpha}=q_{\alpha}^{\prime}-x \hat{g}_{\alpha \beta} z^{\beta}$ and $\hat{g}_{\alpha \beta}(x)$ is the inverse of $\hat{g}^{\alpha \beta}(x)$. The non-singularity condition on $\hat{g}^{\alpha \beta}(x)$ allows us to evaluate the gaussian integral yielding

$$
L(z)=(-i)^{2} \int_{0}^{1} d x \int_{0}^{\infty} d \lambda \lambda i\left(\frac{\pi}{i \lambda}\right)^{n / 2} \frac{1}{\sqrt{-\operatorname{det} \hat{g}^{\alpha \beta}(x)}} e^{x \hat{g}_{\alpha \beta}(x) z^{\alpha} z^{\beta} / 4} e^{-i \lambda m^{2}(1-x)} .
$$

Hence

$$
\begin{aligned}
L(z) & =\frac{i}{(4 \pi)^{n / 2}} \int_{0}^{1} d x \Gamma\left(2-\frac{n}{2}\right)\left(m^{2}(1-x)\right)^{n / 2-2} \frac{1}{\sqrt{-\operatorname{det} \hat{g}^{\alpha \beta}(x)}} e^{i x \hat{g}^{\alpha \beta}(x) z^{\alpha} z^{\beta} / 4} \\
& =-\frac{i}{8 \pi^{2}} \frac{1}{n-4} \int_{0}^{1} d x \frac{1}{\sqrt{-\operatorname{det} \hat{g}^{\alpha \beta}(x)}} e^{i \hat{g}_{\alpha \beta}(x) z^{\alpha} z^{\beta} / 4} .
\end{aligned}
$$

Finally we can reconstruct $\Sigma(0)$ using eq(184).

Similarly we see that

$$
H_{\rho}^{(1) \tau}=i e^{2} \mu^{4-n}\left(\delta_{\rho}^{\mu} \bar{g}^{\tau \nu}+\delta_{\rho}^{\nu} \bar{g}^{\tau \mu}-\delta_{\rho}^{\tau} \bar{g}^{\mu \nu}\right) g_{\mu \lambda}\left(e^{-i \mathbf{N}\left(-i \partial_{z}\right)}\right)^{\lambda}{ }_{\nu} L(z) .
$$

From a slightly more complex calculation we find

$$
H_{\rho}^{(2) \tau}=-2 i e^{2} \bar{g}^{\tau \lambda}\left(\delta_{\rho}^{\mu} \bar{g}^{\sigma \nu}+\delta_{\rho}^{\nu} \bar{g}^{\sigma \mu}-\delta_{\rho}^{\sigma} \bar{g}^{\mu \nu}\right) g_{\mu \kappa}\left(e^{-i \mathbf{N}\left(-i \partial_{z}\right)}\right)^{\kappa}{ }_{\nu} L_{\lambda \sigma}(z) .
$$

where

$$
L_{\lambda \sigma}(z)=-i \int d u \int \frac{d^{n} q}{(2 \pi)^{n}} \frac{q_{\lambda} q_{\sigma}}{\left(\bar{g}^{\alpha \beta} q_{\alpha} q_{\beta}-m^{2}\right)^{2}} e^{i u\left(q^{2}+z \cdot q / \sqrt{u}+i \varepsilon\right)} .
$$

The pole at $n=4$ has the form

$$
L_{\lambda \sigma}(z)=-\frac{1}{8 \pi^{2}} \frac{1}{n-4} \int_{0}^{1} d x \frac{(1-x)}{\sqrt{-\operatorname{det} \hat{g}^{\alpha \beta}(x)}}\left(\frac{i}{2} \hat{g}_{\lambda \sigma}(x)+\frac{x}{4} z^{\alpha} z^{\beta} \hat{g}_{\lambda \alpha}(x) \hat{g}_{\sigma \beta}(x)\right) e^{i x \hat{g}_{\alpha \beta}(x) z^{\alpha} z^{\beta} / 4} \text {. }
$$

\section{Petrov Class of Bimetrically Generated WLTs}

It would be interesting to develop a general theory of how the bimetric structure of the theory affects the nature of Lorentz symmetry breaking through the renormalisation process. At present this seems rather difficult. For now we confine attention to some particular examples involving the simpler Petrov classes. We will adopt a minimal approach that assumes presence of contributions to the WLT $C^{\mu \nu \sigma \tau}$ only of a type forced on us by the need to accomodate the divergences accompanying the WLT $V^{\mu \nu \sigma \tau}$ in section 7 .

\subsection{Class O}

In fact the simplest non-trivial case is class $\mathrm{O}$ for which $V^{\mu \nu \sigma \tau}=0$. There are three cases. They arise when there is a coordinate frame for which $\bar{g}^{\mu \nu}$ has the form

$$
\bar{g}^{\mu \nu}=b g^{\mu \nu} \pm(a-b) k^{\mu} k^{\nu} .
$$

We choose + or - according as $k^{\mu}$ is timelike $\left(g_{\mu \nu} k^{\mu} k^{\nu}>0\right)$ or spacelike $\left(g_{\mu \nu} k^{\mu} k^{\nu}<0\right)$. In order to maintain $\operatorname{det} \bar{g}^{\mu \nu}=-1$ we impose $a b^{3}=1$. There is a third case $k^{\mu}=l^{\mu}$ where the lightlike vector $l^{\mu}$ satisfies $g_{\mu \nu} l^{\mu} l^{\nu}=0$ and

$$
\bar{g}^{\mu \nu}=g^{\mu \nu}+w l^{\mu} l^{\nu} .
$$

It is easy to verify that the WLT $V^{\mu \nu \sigma \tau}$ vanishes in all three cases. In our minimal approach we therefore assume $C^{\mu \nu \sigma \tau}$ vanishes. In the timelike case the underlying reason, of course, is that we are maintaining invariance under the rotation group that leaves $k^{\mu}$ invariant and a WLT cannot exhibit such an invariance unless it is null. Similar remarks apply in the other cases. Under these circumstances although we still have Lorentz symmetry breaking, the lightcone associated with photons being distinct from that associated with electrons, we do not have birefringence for the photons. 


\subsection{Class $\mathrm{N}$}

The next most simple case is class N. Such a Lorentz symmetry breaking situation may be induced by using the NP tetrad to express the electron inverse metric in the form

$$
\bar{g}^{\mu \nu}=g^{\mu \nu}+l^{\mu}\left(\bar{c} m^{\nu}+c \bar{m}^{\nu}\right)+\left(\bar{c} m^{\mu}+c \bar{m}^{\mu}\right) l^{\nu}+\alpha l^{\mu} l^{\nu},
$$

where for the moment $\alpha$ is an arbitrary real parameter. It is readily verified that in this case $\operatorname{det} \bar{g}^{\mu \nu}=-1$ and

$$
V^{\mu \nu \sigma \tau}=\bar{c}^{2} A^{\mu \nu} A^{\sigma \tau}+c^{2} \bar{A}^{\mu \nu} \bar{A}^{\sigma \tau} .
$$

These two cases will be examined in detail later. However a further example provides additional insight into the effect of vacuum polarisation in the bimetric context.

\subsection{Class D}

One simple way of constructing a new metric from the standard one is to consider a shearing of space-time. Such a shearing is represented by the mapping $x^{\mu} \rightarrow x^{\prime \mu}=T^{\mu}{ }_{\nu} x^{\nu}$, where

$$
T_{\nu}^{\mu}=\delta_{\nu}^{\mu}+v f^{\mu} h_{\sigma}
$$

and

$$
\begin{aligned}
h^{2} & =1 \\
f^{2} & =-1 \\
f . h & =0 .
\end{aligned}
$$

We can then define a new metric to be of the form

$$
\bar{g}^{\mu \nu}=T_{\sigma}^{\mu} T^{\nu}{ }_{\tau} \eta^{\sigma \tau} .
$$

If now we calculate $W^{\mu \nu \sigma \tau}=\bar{\gamma}^{\mu \sigma} \bar{\gamma}^{\nu \tau}-\bar{\gamma}^{\mu \tau} \bar{\gamma}^{\nu \sigma}$, we obtain

$$
\begin{aligned}
W^{\mu \nu \sigma \tau}= & \eta^{\mu \sigma} \eta^{\nu \tau}-\eta^{\mu \tau} \eta^{\nu \sigma} \\
& +\eta^{\mu \sigma}\left(v\left(f^{\nu} h^{\tau}+f^{\tau} h^{\nu}\right)+v^{2} f^{\nu} f^{\tau}\right) \\
& +\eta^{\nu \tau}\left(v\left(f^{\mu} h^{\sigma}+f^{\sigma} h^{\mu}\right)+v^{2} f^{\mu} f^{\sigma}\right) \\
& -\eta^{\mu \tau}\left(v\left(f^{\nu} h^{\sigma}+f^{\sigma} h^{\nu}\right)+v^{2} f^{\nu} f^{\sigma}\right) \\
& -\eta^{\nu \sigma}\left(v\left(f^{\mu} h^{\tau}+f^{\tau} h^{\mu}\right)+v^{2} f^{\mu} f^{\tau}\right) \\
& -v^{2}\left(f^{\mu} h^{\nu}-f^{\nu} h^{\mu}\right)\left(f^{\sigma} h^{\tau}-f^{\tau} h^{\sigma}\right) .
\end{aligned}
$$

When we extract $V^{\mu \nu \sigma \tau}$ we obtain

$$
\begin{aligned}
V^{\mu \nu \sigma \tau} \quad & -v^{2}\left\{\frac{1}{3}\left(\eta^{\mu \sigma} \eta^{\nu \tau}-\eta^{\mu \tau} \eta^{\nu \sigma}\right)\right. \\
& +\frac{1}{2} \eta^{\mu \sigma}\left(f^{\nu} f^{\tau}-h^{\nu} h^{\tau}\right) \\
& +\frac{1}{2} \eta^{\nu \tau}\left(f^{\mu} f^{\sigma}-h^{\mu} h^{\sigma}\right) \\
& -\frac{1}{2} \eta^{\mu \tau}\left(f^{\nu} f^{\sigma}-h^{\nu} h^{\sigma}\right) \\
& -\frac{1}{2} \eta^{\nu \sigma}\left(f^{\mu} f^{\tau}-h^{\mu} h^{\tau}\right) \\
& \left.-\left(f^{\mu} h^{\nu}-f^{\nu} h^{\mu}\right)\left(f^{\sigma} h^{\tau}-f^{\tau} h^{\sigma}\right)\right\}
\end{aligned}
$$

Setting $f^{\mu}=\left(l^{\mu}+n^{\mu}\right) / \sqrt{2}$ and $h^{\mu}=\left(l^{\mu}-n^{\mu}\right) / \sqrt{2}$ we find

$$
V^{\mu \nu \sigma \tau}=\frac{1}{6} v^{2}\left\{A^{\mu \nu} B^{\sigma \tau}+B^{\mu \nu} A^{\sigma \tau}+D^{\mu \nu} D^{\sigma \tau}\right\}+\text { c.c.. }
$$


In this case of sheared lightcones we find that the WLT is indeed of Petrov class D. However since the coefficient is real it is not quite the most general case for class D.

The remaining classes, although obviously worth investigating, are considerably more elaborate. For example the case for which $\bar{g}^{\mu \nu}$ is obtained from $g^{\mu \nu}$ by separate rescalings in each of the timelike and spacelike directions leads to a WLT of Petrov class I. We postpone such a completion of our program for a later discussion.

\section{Special Examples of Renormalized Bimetric Theory.}

We look in more detail at renormalisation in two special cases, Petrov classes $\mathrm{O}$ and $\mathrm{N}$ that are particularly tractable.

\subsection{Bimetric Theory with Petrov Class O}

It is convenient to construct the the three metric cases in class $\mathrm{O}$ by introducing a reference metric which we choose to be the standard Lorentz metric $\eta^{\mu \nu}$. For the timelike case we have

$$
\begin{aligned}
g_{0}^{\mu \nu} & =\beta_{0} \eta^{\mu \nu}+\left(\alpha_{0}-\beta_{0}\right) k^{\mu} k^{\nu} \\
g^{\mu \nu} & =\beta \eta^{\mu \nu}+(\alpha-\beta) k^{\mu} k^{\nu} \\
\bar{g}_{0}^{\mu \nu} & =\bar{\beta}_{0} \eta^{\mu \nu}+\left(\bar{\alpha}_{0}-\bar{\beta}_{0}\right) k^{\mu} k^{\nu} \\
\bar{g}^{\mu \nu} & =\bar{\beta} \eta^{\mu \nu}+(\bar{\alpha}-\bar{\beta}) k^{\mu} k^{\nu},
\end{aligned}
$$

where $k^{\mu}=(1,0,0,0)$ and hence $\eta_{\mu \nu} k^{\mu} k^{\nu}=1$. We also find it convenient to set $\bar{\alpha}=a \alpha$ and $\bar{\beta}=b \beta$. Each of the above metrics has a determinant of -1 . In particular we have $\alpha \beta^{3}=\bar{\alpha} \bar{\beta}^{3}=1$. This implies that $a b^{3}=1$. The inverses of the above metrics are easily constructed by the replacements $\alpha \rightarrow \alpha^{-1}$ and $\beta \rightarrow \beta^{-1}$ etc. We have also

$$
\bar{g}^{\mu \nu}=b g^{\mu \nu}+\alpha(a-b) k^{\mu} k^{\nu} .
$$

We then find that

$$
W^{\mu \nu \sigma \tau}=b^{2}\left(g^{\mu \sigma} g^{\nu \tau}-g^{\mu \tau} g^{\nu \sigma}\right)+\alpha(a-b)\left(g^{\mu \sigma} k^{\nu} k^{\tau}+g^{\nu \tau} k^{\mu} k^{\sigma}-g^{\mu \tau} k^{\nu} k^{\sigma}-g^{\nu \sigma} k^{\mu} k^{\tau}\right)
$$

It follows that

$$
V^{\mu \sigma}=\frac{1}{2} b(b-a)\left(g^{\mu \sigma}-4 \alpha k^{\mu} k^{\sigma}\right)
$$

and

$$
W=6 b(a+b)=6\left(b^{2}+b^{-2}\right) .
$$

It is easily confirmed that the WLT $V^{\mu \nu \sigma \tau}$ vanishes. The pole divergence in $C_{0}^{\mu \nu \sigma \tau}$ at $n=4$ therefore also vanishes and we are free to apply our minimal assumption that $C^{\mu \nu \sigma \tau}=0$. In that case the representation of the photon propagator simplifies and we can deduce

$$
\Sigma(0)=-i(i e)^{2} \mu^{4-n} m \bar{g}^{\mu \nu} g_{\mu \nu} L(0)=m \frac{e^{2}}{8 \pi^{2}} \frac{1}{n-4} \bar{g}^{\mu \nu} g_{\mu \nu} \int_{0}^{1} d x \frac{1}{\sqrt{-\operatorname{det} \hat{g}^{\alpha \beta}(x)}},
$$

and

$$
\begin{aligned}
H_{\rho}^{(1) \tau} & =-i(i e)^{2} \mu^{4-n}\left(2 g_{\mu \rho} \bar{g}^{\tau \mu}-\delta_{\rho}^{\tau} \bar{g}^{\mu \nu} g_{\mu \nu}\right) L(0) \\
& =\frac{e^{2}}{8 \pi^{2}}\left(2 g_{\mu \rho} \bar{g}^{\tau \mu}-\delta_{\rho}^{\tau} \bar{g}^{\mu \nu} g_{\mu \nu}\right) \frac{1}{n-4} \int_{0}^{1} d x \frac{1}{\sqrt{-\operatorname{det} \hat{g}^{\alpha \beta}(x)}}
\end{aligned}
$$

From eq(193) we have

$$
\begin{aligned}
H_{\rho}^{(2) \tau} & =2 i(i e)^{2} \mu^{4-n}\left(2 g_{\rho \nu} \bar{g}^{\sigma \nu}-\delta_{\rho}^{\sigma} g_{\mu \nu} \bar{g}^{\mu \nu}\right) \bar{g}^{\tau \beta} L_{\beta \sigma}(0) \\
& =-\frac{e^{2}}{8 \pi^{2}} \frac{1}{n-4}\left(2 g_{\rho \nu} \bar{g}^{\sigma \nu}-\delta_{\rho}^{\sigma} \bar{g}^{\mu \nu} g_{\mu \nu}\right) \bar{g}^{\tau \beta} \int_{0}^{1} d x \frac{1-x}{\sqrt{-\operatorname{det} \hat{g}^{\alpha \beta}(x)}} \hat{g}_{\beta \sigma}(x)
\end{aligned}
$$


Using the explicit form for $g^{\mu \nu}$ and $k^{\mu}$ indicated in subsection 11.1 we have

$$
\bar{g}^{\alpha \beta}=\left(\begin{array}{cccc}
a \alpha & 0 & 0 & 0 \\
0 & -b \beta & 0 & 0 \\
0 & 0 & -b \beta & 0 \\
0 & 0 & 0 & -b \beta
\end{array}\right)
$$

We have then

$$
\hat{g}^{\alpha \beta}(x)=\left(\begin{array}{cccc}
\alpha(a+(1-a) x) & 0 & 0 & 0 \\
0 & -\beta((b+(1-b) x)) & 0 & 0 \\
0 & 0 & -\beta(b+(1-b) x)) & 0 \\
0 & 0 & 0 & -\beta(b+(1-b) x))
\end{array}\right)
$$

The inverse matrix $\hat{g}_{\alpha \beta}(x)$ is obvious and

$$
-\operatorname{det} \hat{g}^{\alpha \beta}(x)=(a+(1-a) x)(b+(1-b) x)^{3} .
$$

We require the integrals

$$
\begin{gathered}
J_{0}=\int_{0}^{1} d x \frac{1}{(a+(1-a) x)^{1 / 2}(b+(1-b) x)^{3 / 2}}=\frac{2}{\sqrt{b}(\sqrt{a}+\sqrt{b})} . \\
J_{1}=\int_{0}^{1} d x \frac{1-x}{(a+(1-a) x)^{3 / 2}(b+(1-b) x)^{3 / 2}}=\frac{2}{\sqrt{a b}(\sqrt{a}+\sqrt{b})^{2}} . \\
J_{2}=\int_{0}^{1} d x \frac{1-x}{(a+(1-a) x)^{1 / 2}(b+(1-b) x)^{5 / 2}}=\frac{2}{3} \frac{(\sqrt{a}+2 \sqrt{b})}{b^{3 / 2}(\sqrt{a}+\sqrt{b})^{2}} .
\end{gathered}
$$

The poles at $n=4$ are then

$$
\begin{gathered}
\Sigma(0)=m \frac{e^{2}}{8 \pi^{2}} \frac{2(a+3 b)}{\sqrt{b}(\sqrt{a}+\sqrt{b})} \frac{1}{n-4} . \\
H_{\rho}^{(1) \tau}=\frac{e^{2}}{8 \pi^{2}}\left(\begin{array}{cccc}
(a-3 b) J_{0} & 0 & 0 & 0 \\
0 & -(a+b) J_{0} & 0 & 0 \\
0 & 0 & -(a+b) J_{0} & 0 \\
0 & 0 & 0 & -(a+b) J_{0}
\end{array}\right) \frac{1}{n-4} . \\
H_{\rho}^{(2) \tau}=-\frac{e^{2}}{8 \pi^{2}}\left(\begin{array}{cccc}
a(a-3 b) J_{1} & 0 & 0 & 0 \\
0 & -b(a+b) J_{2} & 0 & 0 \\
0 & 0 & -b(a+b) J_{2} & 0 \\
0 & 0 & 0 & -b(a+b) J_{2}
\end{array}\right) \frac{1}{n-4} . \\
H^{(1)}=H_{\tau}^{(1) \tau}=-\frac{e^{2}}{8 \pi^{2}} 2(a+3 b) J_{0} \frac{1}{n-4} .
\end{gathered}
$$

The traceless part is

$$
h_{\rho}^{(1) \tau}=H_{\rho}^{(1) \tau}-\frac{1}{4} H^{(1)} \delta_{\rho}^{\tau}=\frac{e^{2}}{8 \pi^{2}} \frac{3}{2}(a-b) J_{0} T_{\rho}^{\tau} \frac{1}{n-4},
$$

where $T_{\rho}^{\tau}$ is the diagonal traceless matrix with diagonal entries $(1,-1 / 3,-1 / 3,-1 / 3)$. We have also

$$
H^{(2)}=H_{\tau}^{(2) \tau}=\frac{e^{2}}{8 \pi^{2}} \frac{4}{n-4} .
$$

The traceless part is

$$
h_{\rho}^{(2) \tau}=-\frac{e^{2}}{8 \pi^{2}} \frac{2 a^{3 / 2}+a \sqrt{b}-4 \sqrt{a} b+b^{3 / 2}}{\sqrt{b}(\sqrt{a}+\sqrt{b})^{2}} T^{\tau} \rho \frac{1}{n-4} .
$$


On combining these results and setting $a=b^{-3}$ we find

$$
H=H^{(1)}+H^{(2)}=-\frac{e^{2}}{8 \pi^{2}} \frac{4\left(2 b^{4}-b^{2}+1\right)}{b^{2}\left(1+b^{2}\right)} \frac{1}{n-4},
$$

and

$$
h_{\rho}^{\tau}=h_{\rho}^{(1) \tau}+h_{\rho}^{(2) \tau}=\frac{e^{2}}{8 \pi^{2}} f(b) T_{\rho}^{\tau} \frac{1}{n-4},
$$

where

$$
f(b)=\frac{\left(1-b^{2}\right)\left(1+3 b^{2}+4 b^{4}\right)}{b^{2}\left(1+b^{2}\right)^{2}} .
$$

Note that $h_{\rho}^{\tau}$ vanishes when $b=1$ as it should since this value corresponds to the restoration of Lorentz symmetry.

\subsubsection{Renormalisation Group for Bimetric Theory - Petrov Class O}

With the above information we can calculate the renormalisation counterterms for the bare parameters of the theory. In the present model $W=6\left(b^{2}+b^{-2}\right)$ with the result that

$$
e_{0}^{2}=\mu^{4-n} e^{2}\left(1-\frac{e^{2}}{12 \pi^{2}} \frac{\left(b^{2}+b^{-2}\right)}{n-4}\right) .
$$

Assuming that the renormalisation process works beyond our second order calculation we can explore the implication of the renormalisation group for this model. Setting $t=\log \left(\mu / \mu_{S}\right)$ where $\mu_{S}$ is a standard scale for which the corresponding renormalised charge, $e_{S}$ is small, we can use the lack of dependence of the bare parameter $e_{0}$ on $\mu$ to deduce that

$$
\frac{d}{d t} e_{0}^{2}=0
$$

It follows then from eq $(228)$ to $O\left(e^{2}\right)$ that

$$
\frac{d}{d t}\left(e^{2}\right)=e^{2}\left(-(4-n)+\frac{e^{2}}{12 \pi^{2}}\left(b^{2}+b^{-2}\right)\right)
$$

The bare metric $g_{0}^{\mu \nu}$ is a diagonal matrix with entries $\left(\alpha_{0}, \beta_{0}, \beta_{0}, \beta_{0}\right)$. We can infer to $O\left(e^{2}\right)$ from eq(175) that

$$
e^{2} g^{(1) \mu \nu}=\frac{e^{2}}{24 \pi^{2}} b(b-a) \frac{1}{n-4}\left(\beta \eta^{\mu \nu}-(\beta+3 \alpha) k^{\mu} k^{\nu}\right)
$$

We find then

$$
\begin{aligned}
& \alpha_{0}=\alpha\left(1-3 \frac{e^{2}}{24 \pi^{2}} b(b-a) \frac{1}{n-4}\right) \\
& \beta_{0}=\beta\left(1+\frac{e^{2}}{24 \pi^{2}} b(b-a) \frac{1}{n-4}\right) .
\end{aligned}
$$

Note that these results are of course consistent with (to $O\left(e^{2}\right)$ ) with the relation $\alpha_{0} \beta_{0}^{3}=1$. Again the bare parameter $\beta_{0}$ is independent of $\mu$ therefore we can conclude that

$$
\frac{d \beta}{d t}=-\beta \frac{e^{2}}{24 \pi^{2}} b(b-a)=-\beta \frac{e^{2}}{24 \pi^{2}}\left(b^{2}-b^{-2}\right) .
$$

From eq(175) we can deduce that

$$
\begin{aligned}
& \bar{\alpha}_{0}=\bar{\alpha}\left(1-\frac{e^{2}}{4 \pi^{2}} f(b) \frac{1}{n-4}\right) \\
& \bar{\beta}_{0}=\bar{\beta}\left(1+\frac{e^{2}}{12 \pi^{2}} f(b) \frac{1}{n-4}\right) .
\end{aligned}
$$


This is consistent with $\bar{\alpha}_{0} \bar{\beta}_{0}^{3}=1$, and we have

$$
\frac{d \bar{\beta}}{d t}=-\bar{\beta} \frac{e^{2}}{12 \pi^{2}} f(b) .
$$

Recalling $\bar{\beta}=b \beta$ we have

$$
\frac{\bar{\beta}_{0}}{\beta_{0}}=b\left(1+\frac{e^{2}}{24 \pi^{2}} F(b) \frac{1}{n-4}\right),
$$

where

$$
F(b)=2 f(b)-\left(b^{2}-b^{-2}\right) .
$$

We obtain the result

$$
\frac{d b}{d t}=-b \frac{e^{2}}{24 \pi^{2}} F(b)
$$

From eq(173) we see that

$$
m_{0}=m\left(1+\frac{e^{2}}{8 \pi^{2}} \frac{4 b^{4}+b^{2}+1}{b^{2}\left(1+b^{2}\right)} \frac{1}{n-4}\right) .
$$

The renormalisation group equation is

$$
\frac{d m}{d t}=-\frac{e^{2}}{8 \pi^{2}} \frac{4 b^{4}+b^{2}+1}{b^{2}\left(1+b^{2}\right)} m .
$$

These RG equations have a particularly significant fixed point at $e^{2}=0$ and $b=1$ which corresponds to the Lorentz invariant case at zero coupling. For small departures from Lorentz invariance, $b=1+y$ where $y$ is small, we find on expanding in powers of $y$ and retaining only linear terms

$$
\frac{d\left(e^{2}\right)}{d t}=\frac{e^{4}}{6 \pi^{2}},
$$

and

$$
\frac{d y}{d t}=3 \frac{e^{2}}{6 \pi^{2}} y \text {. }
$$

The solution for the RG trajectory in the neighbourhood of the fixed point is

$$
\frac{e^{2}}{e_{S}^{2}}=\left(1-\frac{e_{S}^{2}}{6 \pi^{2}} t\right)^{-1}
$$

and

$$
\frac{y}{y_{S}}=\left(1-\frac{e_{S}^{2}}{6 \pi^{2}} t\right)^{-3}
$$

where $e_{S}$ and $y_{S}$ are the assigned values of $e$ and $y$ at $t=0$ or $\mu=\mu_{S}$. This shows that the fixed point is IR attractive and that in its neighbourhood we have

$$
\frac{y}{y_{S}}=\left(\frac{e^{2}}{e_{S}^{2}}\right)^{3} \text {. }
$$

Here $e_{S}^{2}$ and $y_{S}$ are the coupling and (small) departure from Lorentz invariance at the standard scale $\mu=\mu_{S}$. It follows that in the IR limit the theory exhibits the same behaviour as as implied by the analysis in references $[8,5]$. In the same approximation we find from eq(240)

$$
\frac{d m}{d t}=-\frac{3 e^{2}}{8 \pi^{2}} m
$$




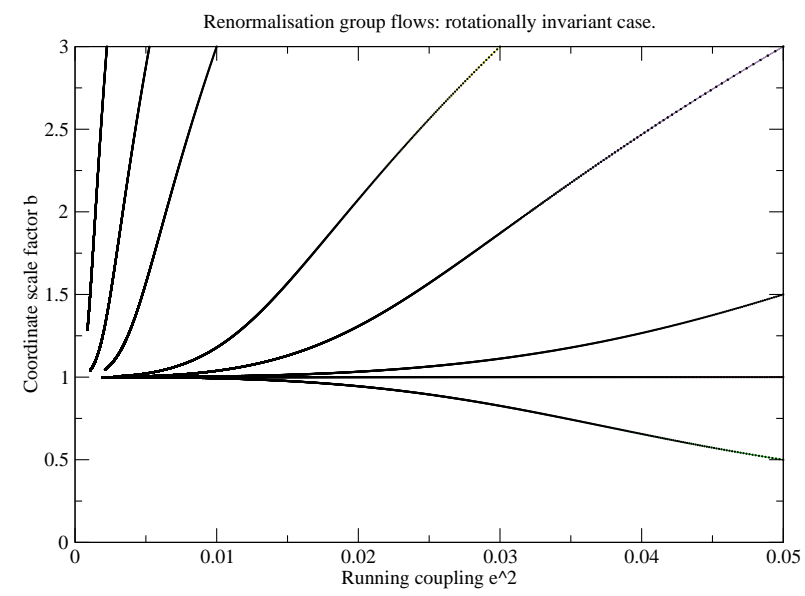

Figure 4: Renormalisation group trajectories Petrov class O: rotationally invariant case.

and therefore we have the result, identical with that for the Lorentz case,

$$
m=m_{S}\left(1-\frac{e_{S}^{2}}{6 \pi^{2}} t\right)^{9 / 4},
$$

where $m_{S}$ is the value of the mass parameter when $\mu=\mu_{S}$.

This gives a description of the behaviour of the effective parameters in the neighbourhood of the point $e^{2}=0, b=1$. However in our approach we can compute the complete RG trajectory without constraint on $b$, provided of course that $e^{2}$ does not become too large. The results are illustrated in Fig.4. An important observation is that no matter how small $e^{2}$ or how large $b$ the RG trajectory never approaches the axis $e^{2}=0$ except at the fixed point discussed above. The axis $e^{2}=0$ is of course a line of fixed points corresponding to a theory with no coupling between electrons and photons. Such a non-interacting theory can maintain any Lorentz symmetry breaking imposed on it. The conclusion is then that no matter how weak the electron-photon coupling or how large the Lorentz symmetry breaking at higher energies the theory will at least in the massless case exhibit Lorentz symmetry in the IR limit as proposed in reference [8].

The case in which the vector $k^{\mu}$ is spacelike results in an essentially identical analysis which need not be repeated explicitly. The case in which $k^{\mu}$ is replaced by a lightlike vector is different in detail but yields essentially similar results, in particular that Lorentz symmetry is restored in the IR limit. It can be viewed as a special case of the model discussed in the next section and we do not treat it separately.

\subsection{Bimetric Theory with Petrov Class N}

The next most tractable example is provided by the choice that $\bar{g}^{\mu \nu}$ has the structure exhibited in eq(196). There is no loss of generality in choosing the parameter $c$ to be real. In order to parametrise the various metrics we introduce in this case a reference metric which we are free to choose to be $\eta^{\mu \nu}$ and an associated NP tetrad $l, n, m, \bar{m}$ with the properties $l^{\mu}=\eta^{\mu \nu} l_{\nu}$, $l^{2}=n^{2}=m^{2}=\bar{m}^{2}=0, l \cdot n=-m \cdot \bar{m}=1$, and $l \cdot m=l \cdot \bar{m}=n \cdot m=n \cdot \bar{m}=0$. We now construct the parametrised metrics in the form

$$
\begin{aligned}
& g_{0}^{\mu \nu}=\eta^{\mu \nu}+s_{0} P^{\mu \nu}+r_{0} l^{\mu} l^{\nu}, \\
& g^{\mu \nu}=\eta^{\mu \nu}+s P^{\mu \nu}+r l^{\mu} l^{\nu},
\end{aligned}
$$




$$
\begin{aligned}
& \bar{g}_{0}^{\mu \nu}=\eta^{\mu \nu}+u_{0} P^{\mu \nu}+v_{0} l^{\mu} l^{\nu}, \\
& \bar{g}^{\mu \nu}=\eta^{\mu \nu}+u P^{\mu \nu}+v l^{\mu} l^{\nu},
\end{aligned}
$$

where

$$
P^{\mu \nu}=l^{\mu}\left(m^{\nu}+\bar{m}^{\nu}\right)+\left(m^{\mu}+\bar{m}^{\mu}\right) l^{\nu} .
$$

Of course the parameters $s, r, u, v$ are the renormalised versions of $s_{0}, r_{0}, u_{0}, v_{0}$ so that $s_{0}=$ $s+e^{2} s^{(1)}$ to $O\left(e^{2}\right)$ where $s^{(1)}$ has a pole at $n=4$ and similarly for the other parameters. We also require an NP tetrad $l(s), m(s), n(s), \bar{m}(s)$ associated with the metric $g^{\mu \nu}$. We achieve this by setting $l_{\mu}(s)=l_{\mu}, m_{\mu}(s)=m_{\mu}$. By imposing the relations $l^{\mu}(s)=g^{\mu \nu} l_{\nu}(s)$ etc, we find

$$
\begin{aligned}
l^{\mu}(s) & =l^{\mu}, \\
m^{\mu}(s) & =m^{\mu}-s l^{\mu}, \\
\bar{m}^{\mu}(s) & =\bar{m}^{\mu}-s l^{\mu} .
\end{aligned}
$$

It is easily checked that $l^{\mu}(s) l_{\mu}(s)=m^{\mu}(s) m_{\mu}(s)=\bar{m}^{\mu}(s) \bar{m}_{\mu}(s)=0$ and $l^{\mu}(s) m_{\mu}(s)=$ $l^{\mu}(s) \bar{m}_{\mu}(s)=0$. In addition $m^{\mu}(s) \bar{m}_{\mu}(s)=-1$. Although we will not make use of it we give for completeness the form of the remaining element of the tetrad thus $n_{\mu}(s)=n_{\mu}-\left(s^{2}+\right.$ $s+r / 2) l_{\mu}-s\left(m_{\mu}+\bar{m}_{\mu}\right)$ and $n^{\mu}(s)=g^{\mu \nu} n_{\nu}(s)=n^{\mu}+\left(s^{2}-s+r / 2\right) l^{\mu}$. It is also easily shown that

$$
g_{\mu \nu}=\eta_{\mu \nu}-s P_{\mu \nu}-\left(2 s^{2}+r\right) l_{\mu} l_{\nu} .
$$

The relation between $g^{\mu \nu}$ and $\bar{g}^{\mu \nu}$ is

$$
\bar{g}^{\mu \nu}-g^{\mu \nu}=c P^{\mu \nu}+w l^{\mu} l^{\nu},
$$

where $c=u-s$ and $w=v-r$. If we define $P^{\mu \nu}(s)=l^{\mu}\left(m^{\nu}(s)+\bar{m}^{\nu}(s)\right)+\left(m^{\mu}(s)+\bar{m}^{\mu}(s)\right) l^{\nu}$ then we have

$$
P^{\mu \nu}(s)=P^{\mu \nu}-4 s l^{\mu} l^{\nu} .
$$

Hence

$$
\bar{g}^{\mu \nu}=g^{\mu \nu}+c P^{\mu \nu}(s)+(w+4 s c) l^{\mu} l^{\nu} .
$$

This is of the same form as eq(196) with the parameter $\alpha=w+4 s c$. The NP tetrad is of course that appropriate to $g^{\mu \nu}$ as constructed here. The result after some algebra is that

$$
V^{\mu \nu \sigma \tau}=c^{2}\left(A^{\mu \nu} A^{\sigma \tau}+\bar{A}^{\mu \nu} \bar{A}^{\sigma \tau}\right),
$$

with $c$ real. Note that strictly speaking we should have used $A^{\mu \nu}(s)=l^{\mu}\left(m^{\nu}(s)+\bar{m}^{\nu}(s)\right)-$ $l^{\nu}\left(m^{\mu}(s)+\bar{m}^{\mu}(s)\right)$ but it is obvious that $A^{\mu \nu}(s)=A^{\mu \nu}$. We also have $W=12$ and

$$
V^{\mu \sigma}=2 c P^{\mu \sigma}(s)+2\left(w+2 s c+c^{2}\right) l^{\mu} l^{\sigma} .
$$

This may also be expressed in the form

$$
V^{\mu \sigma}=2 c P^{\mu \sigma}+2\left(w-2 s c+c^{2}\right) l^{\mu} l^{\sigma} .
$$

From eqs(148) and eq(183) we find here that

$$
\begin{aligned}
& e_{0}^{2}=\mu^{4-n} e^{2}\left(1-\frac{e^{2}}{6 \pi^{2}} \frac{1}{n-4}\right), \\
& s_{0}=s+\frac{e^{2}}{6 \pi^{2}} \frac{c}{n-4}, \\
& r_{0}=r+\frac{e^{2}}{6 \pi^{2}} \frac{\left(w+c^{2}\right)}{n-4} .
\end{aligned}
$$

Again we use the minimalist approach which allows us to write

$$
\begin{aligned}
& C_{0}^{\mu \nu \sigma \tau}=\kappa_{0}\left(A^{\mu \nu} A^{\sigma \tau}+\bar{A}^{\mu \nu} \bar{A}^{\sigma \tau}\right), \\
& C^{\mu \nu \sigma \tau}=\kappa\left(A^{\mu \nu} A^{\sigma \tau}+\bar{A}^{\mu \nu} \bar{A}^{\sigma \tau}\right) .
\end{aligned}
$$


From eq(149) we find

$$
\kappa_{0}=\kappa\left(1-\frac{e^{2}}{6 \pi^{2}} \frac{1}{n-4}\right)+\frac{e^{2}}{6 \pi^{2}} \frac{c^{2}}{n-4} .
$$

In order to discuss the renormalisation of the electron parameters it is necessary to consider the lowest order photon propagator. Although the representation for the photon propagator in eq(111) is useful for exhibiting the pole divergences at $n=4$ in a general context, it is implicitly a power series in the WLT associated with birefringence and the breakdown of Lorentz invariance. In the present case of Petrov class $\mathrm{N}$, it is possible and more convenient to obtain a complete expression for the photon propagator that can be used in perturbation theory calculations. We will choose the gauge so that $\Lambda^{\mu \nu}=g^{\mu \nu}$. In lowest order in $e^{2}$ the inverse photon propagator is given by

$$
M^{\mu \sigma}(q)=\mathcal{M}^{\mu \sigma}(q)=q^{2} g^{\mu \nu}-C^{\mu \nu \sigma \tau} q_{\nu} q_{\tau} .
$$

That is, for Petrov class N,

$$
M^{\mu \sigma}(q)=q^{2} g^{\mu \sigma}-\kappa\left(P^{\mu} P^{\sigma}+\bar{P}^{\mu} \bar{P}^{\sigma}\right),
$$

where

$$
P^{\mu}=A^{\mu \nu} q_{\nu}=l^{\mu} m^{\nu}(s) q_{\nu}-m^{\mu}(s) l^{\nu} q_{\nu}=l^{\mu} m^{\nu} q_{\nu}-m^{\mu} l^{\nu} q_{\nu}
$$

and

$$
\bar{P}^{\mu}=\bar{A}^{\mu \nu} q_{\nu}=l^{\mu} \bar{m}^{\nu}(s) q_{\nu}-\bar{m}^{\mu}(s) l^{\nu} q_{\nu}=l^{\mu} \bar{m}^{\nu} q_{\nu}-\bar{m}^{\mu} l^{\nu} q_{\nu} .
$$

If we set $P_{\mu}=g_{\mu \nu} P^{\nu}$ and $\bar{P}_{\mu}=g_{\mu \nu} \bar{P}^{\nu}$ then

$$
P^{2}=P^{\mu} P_{\mu}=\bar{P}^{2}=\bar{P}^{\mu} \bar{P}_{\mu}=0,
$$

and

$$
P . \bar{P}=P^{\mu} \bar{P}_{\mu}=-(l . q)^{2}=-\left(l^{\mu} q_{\mu}\right)^{2} .
$$

It is then easily verified that the inverse of $M^{\mu \nu}(q)$ is

$$
M_{\mu \nu}(q)=\frac{1}{q^{2}} g_{\mu \nu}+\kappa \frac{\left(P_{\mu} P_{\nu}+\bar{P}_{\mu} \bar{P}_{\nu}\right)}{\left(q^{2}-\kappa(l . q)^{2}\right)\left(q^{2}+\kappa(l . q)^{2}\right)}-\kappa^{2} \frac{(l . q)^{2}\left(P_{\mu} \bar{P}_{\nu}+\bar{P}_{\mu} P_{\nu}\right)}{q^{2}\left(q^{2}-\kappa(l . q)^{2}\right)\left(q^{2}+\kappa(l . q)^{2}\right)} .
$$

This may be rewritten as

$$
M_{\mu \nu}(q)=\frac{1}{q^{2}} g_{\mu \nu}+\kappa \frac{\left(P_{\mu} P_{\nu}+\bar{P}_{\mu} \bar{P}_{\nu}\right)}{\left(g^{(-) \alpha \beta} q_{\alpha} q_{\beta}\right)\left(g^{(+) \alpha \beta} q_{\alpha} q_{\beta}\right)}-\kappa^{2} \frac{(l . q)^{2}\left(P_{\mu} \bar{P}_{\nu}+\bar{P}_{\mu} P_{\nu}\right)}{q^{2}\left(g^{(-) \alpha \beta} q_{\alpha} q_{\beta}\right)\left(g^{(+) \alpha \beta} q_{\alpha} q_{\beta}\right)},
$$

where

$$
g^{( \pm) \alpha \beta}=g^{\alpha \beta} \pm \kappa l^{\alpha} l^{\beta} .
$$

In discussing the divergence structure of the electron propagator we find from eq(160) that

$$
\Sigma(0)=\Sigma^{(1)}+\Sigma^{(2)}+\Sigma^{(3)},
$$

where

$$
\begin{gathered}
\Sigma^{(1)}=i e^{2} m \mu^{4-n} \int \frac{d^{n} q}{(2 \pi)^{n}} \frac{\bar{g}^{\mu \nu} g_{\mu \nu}}{q^{2}\left(\bar{g}^{\alpha \beta} q_{\alpha} q_{\beta}-m^{2}\right)}, \\
\Sigma^{(2)}=i e^{2} m \mu^{4-n} \int \frac{d^{n} q}{(2 \pi)^{n}} \frac{\bar{g}^{\mu \nu}\left(P_{\mu} P_{\nu}+\bar{P}_{\mu} \bar{P}_{\nu}\right)}{\left(g^{(+) \alpha \beta} q_{\alpha} q_{\beta}\right)\left(g^{(-) \alpha \beta} q_{\alpha} q_{\beta}\right)\left(\bar{g}^{\alpha \beta} q_{\alpha} q_{\beta}-m^{2}\right)}, \\
\Sigma^{(3)}=-i e^{2} m \mu^{4-n} \int \frac{d^{n} q}{(2 \pi)^{n}} \frac{(l . q)^{2} \bar{g}^{\mu \nu}\left(P_{\mu} \bar{P}_{\nu}+\bar{P}_{\mu} P_{\nu}\right)}{q^{2}\left(g^{(+) \alpha \beta} q_{\alpha} q_{\beta}\right)\left(\left(g^{(-) \alpha \beta} q_{\alpha} q_{\beta}\right) g^{\alpha \beta} q_{\alpha} q_{\beta}\right)\left(\bar{g}^{\alpha \beta} q_{\alpha} q_{\beta}-m^{2}\right)} .
\end{gathered}
$$

It is easily checked that $\bar{g}^{\mu \nu} g_{\mu \nu}=n, \bar{g}^{\mu \nu} P_{\mu} P_{\nu}=\bar{g}^{\mu \nu} \bar{P}_{\mu} \bar{P}_{\nu}=0$ and $\bar{g}^{\mu \nu} P_{\mu} \bar{P}_{\nu}=-(l . q)^{2}$. We have then

$$
\Sigma^{(1)}=i e^{2} m \mu^{4-n} n I,
$$


where

$$
\begin{gathered}
I=\int \frac{d^{n} q}{(2 \pi)^{n}} \frac{1}{q^{2}\left(\bar{g}^{\alpha \beta} q_{\alpha} q_{\beta}-m^{2}\right)}, \\
\Sigma^{(2)}=0,
\end{gathered}
$$

and

$$
\Sigma^{(3)}=2 i e^{2} m \mu^{4-n} l^{\mu} l^{\nu} l^{\sigma} l^{\tau} I_{\mu \nu \sigma \tau},
$$

where

$$
I_{\mu \nu \sigma \tau}=\int \frac{d^{n} q}{(2 \pi)^{n}} \frac{q_{\mu} q_{\nu} q_{\sigma} q_{\tau}}{q^{2}\left(g^{(+) \alpha \beta} q_{\alpha} q_{\beta}\right)\left(\left(g^{(-) \alpha \beta} q_{\alpha} q_{\beta}\right) g^{\alpha \beta} q_{\alpha} q_{\beta}\right)\left(\bar{g}^{\alpha \beta} q_{\alpha} q_{\beta}-m^{2}\right)} .
$$

In appendix (C) we show that

$$
I \simeq-\frac{i}{8 \pi^{2}} \frac{1}{n-4},
$$

and, making use of results for integrals listed there we can show that

$$
l^{\mu} l^{\nu} l^{\sigma} l^{\tau} I_{\mu \nu \sigma \tau}=0 .
$$

It follows that

$$
\Sigma(0) \simeq \frac{e^{2}}{2 \pi^{2}} m \frac{1}{n-4} .
$$

We have also, referring to eq(164) and eq(165),

$$
H_{\rho}^{(1) \tau}=H_{\rho}^{(11) \tau}+H_{\rho}^{(12) \tau}+H_{\rho}^{(13) \tau}
$$

and

$$
H_{\rho}^{(2) \tau}=H_{\rho}^{(21) \tau}+H_{\rho}^{(22) \tau}+H_{\rho}^{(23) \tau},
$$

where

$$
\begin{gathered}
H_{\rho}^{(11) \tau}=i e^{2} \mu^{4-n}\left(\delta_{\rho}^{\mu} \bar{g}^{\tau \nu}+\delta_{\rho}^{\nu} \bar{g}^{\tau \mu}-\delta_{\rho}^{\tau} \bar{g}^{\mu \nu}\right) g_{\mu \nu} I, \\
H_{\rho}^{(12) \tau}=i e^{2} \mu^{4-n}\left(\delta_{\rho}^{\mu} \bar{g}^{\tau \nu}+\delta_{\rho}^{\nu} \bar{g}^{\tau \mu}-\delta_{\rho}^{\tau} \bar{g}^{\mu \nu}\right) \kappa K_{\mu \nu},
\end{gathered}
$$

where

$$
\begin{gathered}
K_{\mu \nu}=\int \frac{d^{n} q}{(2 \pi)^{n}} \frac{P_{\mu} P_{\nu}+\bar{P}_{\mu} \bar{P}_{\nu}}{\left.\left(g^{(+) \alpha \beta} q_{\alpha} q_{\beta}\right)\left(g^{(-) \alpha \beta} q_{\alpha} q_{\beta}\right)\left(\bar{g}^{\alpha \beta} q_{\alpha} q_{\beta}\right)-m^{2}\right)} . \\
H_{\rho}^{(13) \tau}=-i e^{2} \mu^{4-n}\left(\delta_{\rho}^{\mu} \bar{g}^{\tau \nu}+\delta_{\rho}^{\nu} \bar{g}^{\tau \mu}-\delta_{\rho}^{\tau} \bar{g}^{\mu \nu}\right) \kappa^{2} J_{\mu \nu}
\end{gathered}
$$

with

$$
J_{\mu \nu}=\int \frac{d^{n} q}{(2 \pi)^{n}} \frac{(l . q)^{2}\left(P_{\mu} \bar{P}_{\nu}+\bar{P}_{\mu} P_{\nu}\right)}{q^{2}\left(g^{(+) \alpha \beta} q_{\alpha} q_{\beta}\right)\left(g^{(-) \alpha \beta} q_{\alpha} q_{\beta}\right)\left(\bar{g}^{\alpha \beta} q_{\alpha} q \beta-m^{2}\right)} .
$$

With the aid of integrals evaluated in appendix $\mathrm{C}$ it can be shpown that

$$
K_{\mu \nu} \simeq 0 .
$$

and

$$
J_{\mu \nu} \simeq 0
$$

Hence

$$
H_{\rho}^{(1) \tau} \simeq \frac{e^{2}}{8 \pi^{2}}\left(\delta_{\rho}^{\mu} \bar{g}^{\tau \nu}+\delta_{\rho}^{\nu} \bar{g}^{\tau \mu}-\delta_{\rho}^{\tau} \bar{g}^{\mu \nu}\right) g_{\mu \nu} \frac{1}{n-4} .
$$

We have

$$
H_{\rho}^{(21) \tau}=-2 i e^{2} \mu^{4-n} \bar{g}^{\tau \beta} I_{\beta \sigma}^{\prime}\left(\delta_{\rho}^{\mu} \bar{g}^{\sigma \nu}+\delta_{\rho}^{\nu} \bar{g}^{\sigma \mu}-\delta_{\rho}^{\sigma} \bar{g}^{\mu \nu}\right) g_{\mu \nu},
$$

where

$$
\begin{gathered}
I_{\beta \sigma}^{\prime}=\int \frac{d^{n} q}{(2 \pi)^{n}} \frac{q_{\beta} q_{\sigma}}{q^{2}\left(\bar{g}^{\alpha \beta} q_{\alpha} q_{\beta}-m^{2}\right)^{2}}, \\
H_{\rho}^{(22) \tau}=-2 i e^{2} \kappa \mu^{4-n} \bar{g}^{\tau \beta}\left(\delta_{\rho}^{\mu} \bar{g}^{\sigma \nu}+\delta_{\rho}^{\nu} \bar{g}^{\sigma \mu}-\delta_{\rho}^{\sigma} \bar{g}^{\mu \nu}\right) K_{\alpha \beta \mu \nu}^{\prime},
\end{gathered}
$$


where

$$
\begin{aligned}
K_{\beta \sigma \mu \nu}^{\prime} & =\int \frac{d^{n} q}{(2 \pi)^{n}} \frac{q_{\beta} q_{\sigma}\left(P_{\mu} P_{\nu}+\bar{P}_{\mu} \bar{P}_{\nu}\right)}{\left(g^{(+) \alpha \beta} q_{\alpha} q_{\beta}\right)\left(g^{(-) \alpha \beta} q_{\alpha} q_{\beta}\right)\left(\bar{g}^{\alpha \beta} q_{\alpha} q_{\beta}-m^{2}\right)^{2}}, \\
H_{\rho}^{(23) \tau} & =2 i e^{2} \kappa^{2} \mu^{4-n} \bar{g}^{\tau \beta}\left(\delta_{\rho}^{\mu} \bar{g}^{\sigma \nu}+\delta_{\rho}^{\nu} \bar{g}^{\sigma \mu}-\delta_{\rho}^{\sigma} \bar{g}^{\mu \nu}\right) l^{\xi} l^{\eta} K_{\beta \sigma \xi \eta \mu \nu}^{\prime}
\end{aligned}
$$

where

$$
K_{\beta \sigma \xi \eta \mu \nu}^{\prime}=\int \frac{d^{n} q}{(2 \pi)^{n}} \frac{q_{\beta} q_{\sigma} q_{\xi} q_{\eta}\left(P_{\mu} \bar{P}_{\nu}+\bar{P}_{\mu} P_{\nu}\right)}{q^{2}\left(g^{(+) \alpha \beta} q_{\alpha} q_{\beta}\right)\left(g^{(-) \alpha \beta} q_{\alpha} q_{\beta}\right)\left(\bar{g}^{\alpha \beta} q_{\alpha} q_{\beta}-m^{2}\right)^{2}},
$$

Again using the integrals evaluated in appendix $\mathrm{C}$ it can be shown that $H_{\rho}^{(22) \tau} \simeq H_{\rho}^{(23) \tau} \simeq 0$. Hence

$$
H_{\rho}^{(2) \tau}(0) \simeq-\frac{e^{2}}{4 \pi^{2}}\left(\delta_{\rho}^{\mu} \bar{g}^{\tau \nu}+\delta_{\rho}^{\nu} \bar{g}^{\tau \mu}-\delta_{\rho}^{\tau} \bar{g}^{\mu \nu}\right) g_{\mu \nu} \frac{1}{n-4} .
$$

It follows that

$$
H_{\rho}^{\tau}(0)=-\frac{e^{2}}{8 \pi^{2}}\left(\delta_{\rho}^{\mu} \bar{g}^{\tau \nu}+\delta_{\rho}^{\nu} \bar{g}^{\tau \mu}-\delta_{\rho}^{\tau} \bar{g}^{\mu \nu}\right) g_{\mu \nu} \frac{1}{n-4},
$$

and

$$
H=H_{\tau}^{\tau}(0)=-\frac{e^{2}}{2 \pi^{2}} \frac{1}{n-4} .
$$

We have then

$$
h_{\rho}^{\tau}=-\frac{e^{2}}{4 \pi^{2}}\left(\bar{g}^{\tau \mu} g_{\mu \rho}-\delta_{\rho}^{\tau}\right) \frac{1}{n-4} .
$$

Introducing the expressions for $\bar{g}^{\mu \nu}$ we find

$$
h_{\rho}^{\tau}=\frac{e^{2}}{6 \pi^{2}}\left(c P_{\rho}^{\tau}(s)+(w+4 s c) l^{\tau} l_{\rho}\right) \frac{1}{n-4} .
$$

We find

$$
\left.h_{\rho}^{\mu} \bar{g}^{\rho \nu}=\frac{e^{2}}{6 \pi^{2}} c P^{\mu} \nu+\left(w-2 c^{2}\right)\right) \frac{1}{n-4} .
$$

Recall that $e^{2} g^{(1) \mu \nu}=-h_{\rho}^{\mu} \bar{g}^{\rho \nu}-h_{\rho}^{\nu} \bar{g}^{\rho \mu}$ we find

$$
\begin{aligned}
& u_{0}=u-\frac{e^{2}}{3 \pi^{2}} \frac{c}{n-4} . \\
& v_{0}=v-\frac{e^{2}}{3 \pi^{2}} \frac{w-2 c^{2}}{n-4} .
\end{aligned}
$$

\subsubsection{Renormalisation Group for Bimetric Theory - Petrov Class N}

From eqs (258) using the independence of the bare charge on the renormalisation scale we find

$$
\frac{d e^{2}}{d t}=e^{2}\left((n-4)+\frac{e^{2}}{6 \pi^{2}}\right) .
$$

In 4 dimensions this becomes

$$
\frac{d e^{2}}{d t}=\frac{\left(e^{2}\right)^{2}}{6 \pi^{2}}
$$

with the solution

$$
\frac{e^{2}}{e_{S}^{2}}=\left(1-\frac{e_{S}^{2}}{6 \pi^{2}} t\right)^{-1}
$$

where $e_{S}$ is the value of the coupling when $\mu=\mu_{S}$.

From eqs(258)and eqs(304)e have the result

$$
u_{0}-s_{0}=c\left(1-\frac{e^{2}}{2 \pi^{2}} \frac{1}{n-4}\right) .
$$


Since $u_{0}$ and $s_{0}$ are independent of the renormalisation scale it follows that

$$
\frac{1}{c} \frac{d c}{d t}=\frac{e^{2}}{2 \pi^{2}}
$$

The solution is

$$
\frac{c}{c_{S}}=\left(1-\frac{e_{S}^{2}}{6 \pi^{2}} t\right)^{-3}
$$

$c_{S}$ being the value of the coupling $c$ at the scale $\mu_{S}$. Similarly

$$
v_{0}-r_{0}=w-\frac{e^{2}}{2 \pi^{2}} \frac{w-c^{2}}{n-4},
$$

leading to

$$
\frac{d w}{d t}=\frac{e^{2}}{2 \pi^{2}}\left(w-c^{2}\right)
$$

with the solution

$$
w=\left(w_{S}-\frac{c_{S}^{2}}{2 \pi^{2}}\left(\left(1-\frac{e_{S}^{2}}{6 \pi^{2}} t\right)^{-6}-1\right)\right)\left(1-\frac{e_{S}^{2}}{2 \pi^{2}} t\right)^{-3} .
$$

We see immediately that in the infra red limit $\mu \rightarrow 0$ or $t \rightarrow-\infty$

$$
\begin{aligned}
e^{2} & \rightarrow 0 \\
c & \rightarrow 0 \\
w & \rightarrow 0
\end{aligned}
$$

Hence as we expect the infrared limit is the weak coupling limit and in this limit both $c$ and $w$ vanish bringing the metrics $g^{\mu \nu}$ and $\bar{g}^{\mu \nu}$ into coincidence thus potentially removing the breakdown of Lorentz invariance, at least in the massless case. We have also from eq(260)

$$
\frac{d \kappa}{d t}=\frac{e^{2}}{6 \pi^{2}} \kappa-\frac{e^{2}}{6 \pi^{2}} c^{2}
$$

Using the above results we have

$$
\kappa=\left(\kappa_{S}-\frac{c_{S}^{2}}{5}\left(\left(1-\frac{e_{S}^{2}}{6 \pi^{2}} t\right)^{-5}-1\right)\right)\left(1-\frac{e_{S}^{2}}{6 \pi^{2}} t\right)^{-1} .
$$

It follows that in the infrared limit $\kappa$ vanishes and hence any birefringence.

Note that it we can choose $c_{S}=\kappa_{S}=0$ which implies $c=\kappa=0$ and therefore we can consistently set $s=u=\kappa=0$. This leaves $w$ as the only significant remaining variable which provides a Lorentz breaking scenario that is the lightlike case of Petrov class O referred to in the previous section. Were we then to choose $w_{S}=0$ we would return to a situation of Lorentz invariance. If however $c_{S} \neq 0$ then we induce nonvanishing values for $w$. For the massless case we can again argue that Lorentz invariance returns in the infrared limit. However if we examine the renormalisation group for the mas we find

$$
m=m_{S}\left(1-\frac{e_{S}^{2}}{6 \pi^{2}} t\right)^{9 / 4} .
$$

This is the same behaviour as the Petrov class 0 model in the neighbourhood of the IR fixed point. However in this case it holds for finite values of the Lorentz breaking parameters. Again we require a closer examination of the infrared limit in this case to deal with a non-zero mass for the electron. 


\section{Conclusions}

We have examined the breakdown of Lorentz invariance in QED through a premetric formulation of elctrodynamics parametrised by a tensor $U^{\mu \nu \sigma \tau}$ that has the same symmetry properties as the Riemann tensor. However we showed that in fact there is a preferred metric $g^{\mu \nu}$ that allows us to decompose $U^{\mu \nu \sigma \tau}$ in the form

$$
U^{\mu \nu \sigma \tau}=g^{\mu \sigma} g^{\nu \tau}-g^{\mu \tau} g^{\nu \sigma}-C^{\mu \nu \sigma \tau}
$$

where $C^{\mu \nu \sigma \tau}$ has the symmetry properties of the Weyl tensor. We can therefore use the Petrov classification for $C^{\mu \nu \sigma \tau}$ to delineate all the possible forms of Lorentz symmetry breaking in electrodynamics and ultimately in QED. Apart from the null case for which there is no Lorentz symmetry breaking in electrodynamis (QED requires further analyis) all the other canonical examples exhibit birefringence. We established the dispersion relations for each Petrov class. In all cases this has the form of homogeneous quartic constraint on the wave vector of the mode. In some cases this quartic has two quadratic factors each corresponding to a particular polarisation. Each factor yields a separate and distinct light cone. In other cases the quartic does not factorise in this way and hence is inherently more complex than a simple double lightcone structure.

In examining the plane wave solutions of the general Lorentz symmetry breaking case we made use of the gauge condition on the elctromagnetic field $g^{\mu \nu} \partial_{\mu} A_{\nu}(x)=0$. However, motivated by the potential absence of Lorentz symmetry we also explored a more general gauge condition $\Lambda^{\mu \nu} \partial_{\mu} A_{\nu}(x)=0$. The choice of gauge condiditon does not affect the physical solutions but it does affect the unphysical ones. In fact $\Lambda^{\mu \nu}$ determines the light cone for these unphysical modes and also for the associated ghost modes. The latter do not play any role in electrodynamics or QED but in a non-abelian gauge theory they will do. In fact it turns out that the more general gauge condition comes into its own when we consider the renormalisation program for QED.

We examine the general structure of renormalised BIMQED to one loop order in perturbation theory but without assuming that the Lorentz symmetry breaking is itself small. The nature of this breaking is determined by the metric $\bar{g}^{\mu \nu}$ governing the propagation of the electron field through the tensor $W^{\mu \nu \sigma \tau}=\bar{g}^{\mu \sigma} \bar{g}^{\nu \tau}-\bar{g}^{\mu \tau} \bar{g}^{\nu \sigma}$ which appears as a factor in the residue of the UV divergence of the vacuum polarisation diagram. The standard decomposition of this tensor leads to a traceless Weil-like tensor $V^{\mu \nu \sigma \tau}$. The Petrov class of this tensor can be used to determine the nature of the Lorentz symmetry breaking in the model. We give examples, though by no means a complete list, of how different choices for $\bar{g}^{\mu \nu}$ lead to different Petrov classes for $V^{\mu \nu \sigma \tau}$.

Finally we apply the renormalisation program in detail to the two simplest Petrov classes of symmetry breaking, namely class $\mathrm{O}$ and class $\mathrm{N}$. We derive the renormalisation group flows in these cases and conclude that Lorentz symmetry breaking is suppressed in the infra-red limit at least in the massless case. The results are entirely consistent with previous analyses. In our case we are not restricted to small deviations and can show, to $O\left(e^{2}\right)$, that the result holds however large the breaking at shorter distances. That is there appear to be no unexpected fixed points for nonvanishing Lorentz symmetry breaking.

It is of course of great interest to examine the corresponding case of a non-abelian gauge theory such as QCD where the weak coupling fixed point occurs in the ultraviolet rather than the infrared limit. We will consider this case in a later paper.

\section{A Gauge Fixed Action for the EM Field}

The partition function for the electromagnetic field is $Z$ given by

$$
Z=\int d[A] e^{i S_{(p)}[A]} .
$$

However $S_{(p)}[A]$ is invariant under the gauge transformation $A_{\mu}(x) \rightarrow A_{\mu}^{h}(x)=A_{\mu}(x)+\partial_{\mu} h(x)$. The expression for $Z$ contains therefore a factor $\int d[h]$ which we wish to extract. In anticipation 
of the gauge condition we wish to invoke namely

$$
\Lambda^{\mu \nu} \partial_{\mu} A_{\nu}(x)=0
$$

we use the $\delta$-functional identity

$$
\int d[h] \Delta \delta\left[C(x)-\Lambda^{\mu \nu} \partial_{\mu} A_{\nu}^{h}(x)\right]=1
$$

where

$$
\Delta=\operatorname{det} \frac{\delta}{\delta h\left(x^{\prime}\right)}\left(\Lambda^{\mu \nu} \partial_{\mu} A_{\nu}^{h}(x)\right)=\operatorname{det} \Lambda^{\mu \nu} \partial_{\mu} \partial_{\nu} \delta\left(x-x^{\prime}\right) .
$$

We now rewrite $Z$ as

$$
Z=\int d[h] \int d[A] \Delta \delta\left[C(x)-\Lambda^{\mu \nu} \partial_{\mu} A_{\nu}^{h}(x)\right] e^{i S_{(p)}[A]} .
$$

Exploiting the gauge invariance of $S_{(p)}[A]$ and the measure $d[A]$, we can write this in the form

$$
Z=\int d[h] \int d[A] \Delta \delta\left[C(x)-\Lambda^{\mu \nu} \partial_{\mu} A_{\nu}(x)\right] e^{i S_{(p)}[A]} .
$$

Because it is a constant we can now drop the factor $\int d[h]$ in the above expression for $Z$. We could also drop the factor $\Delta$ since for QED, it does not depend on the fields in the integrand. However we retain it in order to elucidate the BRST transormation and to anticipate the corresponding results for non-Abelian gauge theories. To this end we introduce anti-commuting ghost fields $c(x)$ and $\bar{c}(x)$ and express $\Delta$ (up to an irrelevant constant factor) in the form

$$
\begin{aligned}
\Delta & =\int d[c] d[\bar{c}] \exp \left\{i \int d^{n} x \Omega \bar{c}(x) \Lambda^{\mu \nu} \partial_{\mu} \partial_{\nu} c(x)\right\} \\
& =\int d[c] d[\bar{c}] \exp \left\{-i \int d^{n} x \Omega \partial_{\mu} \bar{c}(x) \Lambda^{\mu \nu} \partial_{\nu} c(x)\right\}
\end{aligned}
$$

The second equality results from an integration by parts in the exponent. Using arguments we are also free to replace $Z$ in eq(323) by

$$
\begin{aligned}
Z & =\int d[C] \exp \left\{-\frac{i}{2} \int d^{n} x C(x)^{2}\right\} \int d[A] \Delta \delta\left[C(x)-\Lambda^{\mu \nu} \partial_{\mu} A_{\nu}(x)\right] e^{i S_{(p)}[A]} \\
& =\int d[A] d[c] d[\bar{c}] \exp \left\{i S_{\text {g.f. }}\right\}
\end{aligned}
$$

where $S_{\text {g.f. }}$ is the full gauge fixed action for the photon sector given by

$$
S_{\text {g.f. }}=\int d^{n} x\left\{-\frac{1}{4} g^{\mu \sigma} g^{\nu \tau} F_{\mu \nu} F_{\sigma \tau}-\frac{1}{2} \Lambda^{\mu \nu} \Lambda^{\sigma \tau} \partial_{\mu} A_{\nu}(x) \partial_{\sigma} A_{\tau}(x)-\partial_{\mu} \bar{c}(x) \Lambda^{\mu \nu} \partial_{\nu} c(x)\right\} .
$$

The equation of motion for the photon field is

$$
g^{\mu \sigma} g^{\nu \tau} \partial_{\mu} \partial_{\sigma} A_{\nu}-\left(g^{\mu \sigma} g^{\nu \tau}-\Lambda^{\mu \sigma} \Lambda^{\nu \tau}\right) \partial_{\nu} \partial_{\sigma} A_{\mu}=0,
$$

and those for the ghost fields are

$$
\begin{aligned}
& \Lambda^{\mu \nu} \partial_{\mu} \partial_{\nu} c(x)=0 \\
& \Lambda^{\mu \nu} \partial_{\mu} \partial_{\nu} \bar{c}(x)=0 .
\end{aligned}
$$

Clearly $\Lambda^{\mu \nu}$ does play the role of the (inverse) metric for the ghost fields. 


\section{B Removal of Spurious Singularity}

We can verify the absence of the apparent singularity at $q^{2}=0$ by first introducing $\hat{q}$ as the parity reflection of $q$. Of course $\hat{q}^{2}=q^{2}$ and $q . \hat{q}>0$ for $q \neq 0$. We then define the matrix $\hat{\mathcal{M}}^{\nu \tau}$ given by

$$
\hat{\mathcal{M}}^{\nu \tau}=\left(\delta_{\rho}^{\nu}+\frac{\hat{q}^{\nu} q_{\rho}}{q^{2}}\right) \mathcal{M}^{\rho \lambda}\left(\delta_{\lambda}^{\tau}+\frac{q_{\lambda} \hat{q}^{\tau}}{q^{2}}\right) .
$$

That is

$$
\hat{\mathcal{M}}^{\nu \tau}=\mathcal{M}^{\nu \tau}+q^{\nu} \hat{q}^{\tau}+\hat{q}^{\nu} q^{\tau}+\hat{q}^{\nu} \hat{q}^{\tau} .
$$

It follows that $\operatorname{det} \hat{\mathcal{M}}^{\nu \tau}$ is finite and in general nonvanishing on $q^{2}=0$. From eq(330) we find

$$
\mathcal{M}^{\rho \lambda}=\left(\delta_{\nu}^{\rho}-\frac{\hat{q}^{\rho} q_{\nu}}{q^{2}+\hat{q} \cdot q}\right) \hat{\mathcal{M}}^{\nu \tau}\left(\delta_{\tau}^{\lambda}-\frac{q_{\tau} \hat{q}^{\lambda}}{q^{2}+q \cdot \hat{q}}\right) .
$$

From eq(331) we obtain

$$
\mathcal{M}_{\rho \lambda}=\left(\delta_{\rho}^{\nu}+\frac{q_{\rho} \hat{q}^{\nu}}{q^{2}}\right) \hat{\mathcal{M}}_{\nu \tau}\left(\delta_{\lambda}^{\tau}+\frac{\hat{q}^{\tau} q_{\lambda}}{q^{2}}\right)
$$

where $\hat{\mathcal{M}}_{\nu \tau}$ is the inverse of $\hat{\mathcal{M}}^{\nu \tau}$. It is then straightforward to express eq(92) in the form

$$
M_{\nu \tau}=\left(\delta_{\nu}^{\rho}-\frac{q_{\nu} Q^{\rho}}{Q . q}\right) \hat{\mathcal{M}}_{\rho \lambda}\left(\delta_{\tau}^{\lambda}-\frac{Q^{\lambda} q_{\tau}}{Q . q}\right)+\frac{q_{\nu} q_{\tau}}{(Q . q)^{2}} .
$$

Eq(333) shows clearly that $M_{\nu \tau}$ is singular only on the ghost mass-shell and the surface yielding the dipersion relations for the physical states.

\section{Special Integrals}

We will need the following integrals

$$
\begin{aligned}
I & =\int \frac{d^{n} q}{(2 \pi)^{n}} \frac{1}{q^{2}\left(\bar{g}^{\alpha \beta} q_{\alpha} q_{\beta}-m^{2}\right)} . \\
I_{\mu \nu}^{\prime} & =\int \frac{d^{n} q}{(2 \pi)^{n}} \frac{q_{\mu} q_{\nu}}{q^{2}\left(\bar{g}^{\alpha \beta} q_{\alpha} q_{\beta}-m^{2}\right)^{2}} . \\
I_{\mu \nu} & =\int \frac{d^{n} q}{(2 \pi)^{n}} \frac{q_{\mu} q_{\nu}}{\left(g^{(+) \alpha \beta} q_{\alpha} q_{\beta}\right)\left(g^{(-) \alpha \beta} q_{\alpha} q_{\beta}\right)\left(\bar{g}^{\alpha \beta} q_{\alpha} q_{\beta}-m^{2}\right)} . \\
I_{\mu \nu \sigma \tau}^{\prime} & =\int \frac{d^{n} q}{(2 \pi)^{n}} \frac{q_{\mu} q_{\nu} q_{\sigma} q_{\tau}}{\left(g^{(+) \alpha \beta} q_{\alpha} q_{\beta}\right)\left(g^{(-) \alpha \beta} q_{\alpha} q_{\beta}\right)\left(\bar{g}^{\alpha \beta} q_{\alpha} q_{\beta}-m^{2}\right)^{2}} . \\
I_{\mu \nu \sigma \tau} & =\int \frac{d^{n} q}{(2 \pi)^{n}} \frac{q_{\mu} q_{\nu} q_{\sigma} q_{\tau}}{q^{2}\left(g^{(+) \alpha \beta} q_{\alpha} q_{\beta}\right)\left(g^{(-) \alpha \beta} q_{\alpha} q_{\beta}\right)\left(\bar{g}^{\alpha \beta} q_{\alpha} q_{\beta}-m^{2}\right)} . \\
I_{\mu \nu \sigma \tau \xi \eta}^{\prime} & =\int \frac{d^{n} q}{(2 \pi)^{n}} \frac{q_{\mu} q_{\nu} q_{\sigma} q_{\tau} q_{\xi} q_{\eta}}{q^{2}\left(g^{(+) \alpha \beta} q_{\alpha} q_{\beta}\right)\left(g^{(-) \alpha \beta} q_{\alpha} q_{\beta}\right)\left(\bar{g}^{\alpha \beta} q_{\alpha} q_{\beta}-m^{2}\right)^{2}} .
\end{aligned}
$$

\section{C.1 I}

The first integral we study is $I$. It can be put in the form

$$
I=(-i)^{2} \int_{0}^{\infty} d u \int_{0}^{\infty} d v \int \frac{d^{n} q}{(2 \pi)^{n}} \exp \left\{i u\left(g^{\alpha \beta} q_{\alpha} q_{\beta}+i \varepsilon\right)+i v\left(\bar{g}^{\alpha \beta} q_{\alpha} q_{\beta}-m^{2}+i \varepsilon\right)\right\} .
$$

If we change variables so that $u=x \lambda$ and $v=(1-x) \lambda$ we find

$$
I=(-i)^{2} \int_{0}^{1} d x \int_{0}^{\infty} d \lambda \lambda \int \frac{d^{n} q}{(2 \pi)^{n}} \exp \left\{\hat{g}^{\alpha \beta} q_{\alpha} q_{\beta}-(1-x) m^{2}+i \varepsilon\right\},
$$


where

$$
\hat{g}^{\alpha \beta}=x g^{\alpha \beta}+(1-x) \bar{g}^{\alpha \beta} .
$$

That is

$$
\hat{g}^{\alpha \beta}=g^{\alpha \beta}+c(1-x) P^{\alpha \beta}(s)+\alpha(1-x) l^{\alpha} l^{\beta} .
$$

It follows that $\operatorname{det} \hat{g}^{\alpha \beta}=-1$. In this case the interpolating metric $\hat{g}^{\alpha \beta}$ never becomes singular. The constraints of causality as elucidated in ref [] are automatically satisfied. The same will be true of the generalised interpolating matrices encountered below. On performing the $d^{n} q$ integral we find

$$
I=\frac{i}{(4 \pi)^{n / 2}} \int_{0}^{1} d x \int_{0}^{\infty} d(i \lambda)(i \lambda)^{1-n / 2} \exp \left\{-i \lambda\left[(1-x) m^{2}-i \varepsilon\right]\right\} .
$$

That is

$$
I=\frac{i}{(4 \pi)^{n / 2}} \int_{0}^{1} d x \Gamma(2-n / 2)\left[(1-x) m^{2}-i \varepsilon\right]^{n / 2-2} .
$$

The pole at $n=4$ is then

$$
I \simeq-\frac{i}{8 \pi^{2}} \frac{1}{n-4}
$$

\section{C.2 $I_{\mu \nu}^{\prime}$}

Following the pattern of the previous calculation with appropriate changes to accommodate the changed powers in the denominator we can put the integral in the form

$$
I_{\mu \nu}^{\prime}=i \int d x(1-x) \int d \lambda \lambda^{2} \int \frac{d^{n} q}{(2 \pi)^{n}} q_{\mu} q_{\nu} \exp \left\{i \lambda \hat{g}^{\alpha \beta} q_{\alpha} q_{\beta}\right\} \exp \left\{-i \lambda(1-x) m^{2}-i \varepsilon\right\} .
$$

Here $\hat{g}^{\alpha \beta}$ is the same as in the prvious example. The invariance properties of the integral allow us to make the replacement

$$
q_{\mu} q_{\nu} \exp \left\{i \lambda \hat{g}^{\alpha \beta} q_{\alpha} q_{\beta}\right\} \rightarrow \frac{1}{n} \hat{g}_{\mu \nu}\left(\hat{g}^{\alpha \beta} q_{\alpha} q_{\beta}\right) \exp \left\{i \lambda \hat{g}^{\alpha \beta} q_{\alpha} q_{\beta}\right\}=\frac{1}{n} \hat{g}_{\mu \nu} \frac{\partial}{\partial(i \lambda)} \exp \left\{i \lambda \hat{g}^{\alpha \beta} q_{\alpha} q_{\beta}\right\}
$$

We then have after performing the $d^{n} q$ and $d \lambda$ integrations

$$
I_{\mu \nu}^{\prime}=\frac{i}{2(4 \pi)^{n / 2}} \int d x(1-x) \hat{g}_{\mu \nu} \Gamma(2-n / 2)\left[(1-x) m^{2}-i \varepsilon\right]^{n / 2-2},
$$

where

$$
\hat{g}_{\mu \nu}=g_{\mu \nu}-c(1-x) P_{\mu \nu}(s)-\left((1-x) \alpha+2(1-x)^{2} c^{2}\right) l_{\mu} l_{\nu} .
$$

We have then

$$
I_{\mu \nu}^{\prime} \simeq-\frac{i}{16 \pi^{2}} \int d x(1-x) \hat{g}_{\mu \nu} \frac{1}{n-4}
$$

Substituting the expression for $\hat{g}_{\mu \nu}$ we obtain finally

$$
I_{\mu \nu}^{\prime}=-\frac{i}{32 \pi^{2}}\left\{g_{\mu \nu}-\frac{2}{3} c P_{\mu \nu}(s)-\left(\frac{2}{3} \alpha+c^{2}\right) l_{\mu} l_{\nu}\right\} .
$$

\section{C.3 $I_{\mu \nu}$}

We can express each propagator as before and obtain the representation

$$
I_{\mu \nu}=(-i)^{3} \int d u d v d w \int \frac{d^{n} q}{(2 \pi)^{n}} q_{\mu} q_{\nu} \exp \left\{u\left(g^{(+) \alpha \beta} q_{\alpha} q_{\beta}\right)+v\left(g^{(-) \alpha \beta} q_{\alpha} q_{\beta}\right)+w\left(\bar{g}^{\alpha \beta} q_{\alpha} q_{\beta}-m^{2}\right)\right\} .
$$


Using the transformation of integration variables $u=\lambda x, v=\lambda y, w=\lambda z$ we obtain

$$
I_{\mu \nu}=i \int d x d y d z \delta(1-x-y-z) \int d \lambda \lambda^{2} \int \frac{d^{n} q}{(2 \pi)^{n}} q_{\mu} q_{\nu} \exp \left\{i \hat{g}^{\alpha \beta} q_{\alpha} q_{\beta}\right\} \exp \left\{-i \lambda\left[z m^{2}-i \varepsilon\right]\right\}
$$

where now

$$
\hat{g}^{\alpha \beta}=g^{\alpha \beta}+z c P^{\alpha \beta}(s)+(\kappa(x-y)+\alpha z) l^{\alpha} l^{\beta} .
$$

We have again $\operatorname{det} \hat{g}^{\alpha \beta}=-1$ and the inverse matrix is

$$
\hat{g}_{\alpha \beta}=g_{\alpha \beta}-z c P_{\alpha \beta}(s)-\left(\kappa(x-y)+\alpha z+2 c^{2} z^{2}\right) l_{\alpha} l_{\beta} .
$$

As in the previous calculation we we make the substitution $q_{\mu} q_{\nu} \rightarrow \hat{g}_{\mu \nu}\left(\hat{g}^{\alpha \beta} q_{\alpha} q_{\beta}\right) / n$ and obtain after performing the $d^{n} a$ and $d \lambda$ integrations

$$
I_{\mu \nu} \simeq-\frac{i}{(4 \pi)^{2}} \int d x d y d z \delta(1-x-y-z) \hat{g}_{\mu \nu} \frac{1}{n-4}
$$

Finally

$$
I_{\mu \nu}=-\frac{i}{32 \pi^{2}}\left\{g_{\mu \nu}-\frac{1}{3} c P_{\mu \nu}(s)-\frac{1}{3}\left(\alpha+c^{2}\right) l_{\mu} l_{\nu}\right\} \frac{1}{n-4}
$$

\section{C.4 $I_{\mu \nu \sigma \tau}^{\prime}$}

With mild generalisations we can use the techniques of the previous calculations to obtain the result

$$
I_{\mu \nu \sigma \tau}^{\prime} \simeq-\frac{i}{32 \pi^{2}} \int d x d y d z \delta(1-x-y-z) z\left(\hat{g}_{\mu \nu} \hat{g}_{\sigma \tau}+\cdots\right) \frac{1}{n-4},
$$

where $\hat{g}_{\mu \nu}$ is the same as in the previous calculation, and $\left(\hat{g}_{\mu \nu} \hat{g}_{\sigma \tau}+\cdots\right)=\left(\hat{g}_{\mu \nu} \hat{g}_{\sigma \tau}+\hat{g}_{\mu \sigma} \hat{g}_{\nu \tau}+\right.$ $\left.\hat{g}_{\mu \tau} \hat{g}_{\sigma \nu}\right)$.

\section{C.5 $I_{\mu \nu \sigma \tau}$}

Again we can use the same style of calculation to obtain

$$
I_{\mu \nu \sigma \tau} \simeq=-\frac{i}{32 \pi^{2}} \int d x d y d z d t \delta(1-x-y-z-t)\left(\hat{g}_{\mu \nu} \hat{g}_{\sigma \tau}+\cdots\right) \frac{1}{n-4} .
$$

Here

$$
\hat{g}_{\mu \nu}=g_{\mu \nu}-2 t c\left(+l_{\mu}\left(m_{\nu}(s)+\bar{m}_{\nu}(s)\right)+\left(m_{m} u(s)+\bar{m}_{\mu}(s)\right) l_{\nu}\right)-(\kappa(y-z)-t \alpha) l_{\mu} l_{\nu} .
$$

\section{C.6 $I_{\mu \nu \sigma \tau \xi \eta}^{\prime}$}

Finally we obtain using the above style of calkculation

$$
I_{\mu \nu \sigma \tau \xi \eta}^{\prime} \simeq-\frac{i}{64 \pi^{2}} \int d x d y d z d t \delta(1-x-y-z-t) t\left(\hat{g}_{\mu \nu} \hat{g}_{\sigma \tau} \hat{g}_{\xi \eta}+\cdots\right) \frac{1}{n-4} .
$$

Here $\hat{g}_{\mu \nu}$ is the same as in the previous calculation and $\left(\hat{g}_{\mu \nu} \hat{g}_{\sigma \tau} \hat{g}_{\xi \eta}+\cdots\right)$ is a sum of 15 terms that symmetrise the exhibited term with respect to the labels $\{\mu \nu \ldots \eta\}$.

It is easy now to check that for each of the interpolating metrics above we have

$$
l^{\alpha} \hat{g}_{\alpha \beta}=l_{\beta},
$$

and making use of this result we obtain the null results indicated in section 12.2. 


\section{References}

[1] I. T. Drummond. Quantum field theory in a multimetric background. Phys. Rev. D, 88:025009, 2013.

[2] D. Collady and V. A. Kostalecký. Cpt violation and the standard model. Phys. Rev. D, 55:6760, 1997.

[3] D. Collady and V. A. Kostalecký. Lorentz-violating extension of the standard model. Phys. Rev. D, 58:116002, 1998.

[4] V. A. Kostelecký. Data tables for Lorentz and CPT violation. Rev. Mod. Phys., 83:11, 2011.

[5] C. D. Lane V. A. Kostelecký and A. G. M. Pickering. One-loop renormalization of Lorentzviolating electrodynamics. Phys. Rev. D, 65:056006, 2002.

[6] S. Coleman and S. Glashow. High-energy tests of Lorentz invariance. Phys. Rev. D, 59:116008-1, 1999.

[7] S. Coleman and S. L. Glashow. Cosmic ray and neutrino tests of special relativity. Phys. Letts. B, 405:249, 1997.

[8] H. B. Nielsen and M. Ninomiya. $\beta$-function in a non-covariant Yang-Mills theory. Nucl. Phys. B, 141:153, 1978.

[9] H. B. Nielsen and I. Picek. Lorentz non-invariance. Nucl. Phys. B, 211:269, 1983.

[10] S. Chada and H. B. Nielsen. Lorentz invariance as a low energy phenomenon. Nocl. Phys. $B, \mathbf{2 1 7}: 125,1983$.

[11] Y. Itin. On light propagation in premetric electrodynamics. J. Phys. A: Math. Theor., 42:475402, 2009.

[12] A. Z. Petrov. Classification of spaces defined by gravitational fields. Uch. Zapiski Kazan Gos. Univ., 144:55, 1954.

[13] F. R. Klinkhamer and M. Schreck. Consistency of isotropic Maxwell theory:microcausality an unitarity. Nucl. Phys. B, 848:90-107, 2011.

[14] M. Schreck. Quantum field theory based on birefringent modified Maxwell theory. Phys. Rev. D, 89:085013, 2014.

[15] M. Cambiaso, R. Lehnert, R. Potting. Asymptotic states and renormalisation in Lorentzviolating quantum field thoery. Phys. Rev. D, 90:065003, 2014.

[16] I. T. Drummond and S. J. Hathrell. QED vacuum polarization in a background gravitational field and its effect on the velocity of photons. Phys. Rev. D, 22:343, 1980.

[17] T. J. Hollowood and G. M. Shore. Refractive index of curved spacetime:the fate of causality in QED. Nucl. Phys. B, 795:138, 2008.

[18] T. J. Hollowood and G. M. Shore. The effect of gravitational tidal forces on vacuum polarisation: How to undress a photon. Phys. Lett. B, 691:279, 2010.

[19] T. J. Hollowood and G. M. Shore. The unbearable beingness of light, dressing and undressing photons in black hole spacetimes. Int. J. Mod. Phys. D, 21:1241003, 2012.

[20] E. T. Newman and R. Penrose. An approach to gravitational radiation by a method of spin coefficients. J. Math. Phys., 3:566, 1962.

[21] J. Stewart. Advanced General Relativity. Cambridge Monographs on Mathematical Physics, Cambridge University Press, 1991.

[22] R. Penrose and W. Rindler. Spinors and spacetime 2: Spinor and twistor methods in spacetime geometry. Cambrige University Press, Cambridge, 1986.

[23] P. O'Donnell. Introduction to 2-spinors in General Relativity. World Scientific Publishing Co. Pte. Ltd., 2003. 